\title{
Stabilization of Needle-Crystals by the Gibbs-Thomson Effect
}

\author{
C.-A. Pillet ${ }^{\star}$ \\ Département de Physique Théorique, Université de Genève, CH-1211 Geneva 4, Switzerland
}

Received March 16, 1990

\begin{abstract}
We develop a scheme based on pseudo-differential operators to analyze the propagation of excitations in inhomogeneous extended systems. This method is used in a very specific situation, however we think that it has some generality and should apply to various other problems of current interest. We study the well known two-dimensional symmetric model of solidification introduced by Langer and Turski. Assuming the existence of Ivantsov-like steady-state solutions, we calculate their excitation spectrum. We show that there are no unstable propagating modes if the Gibbs-Thomson effect is taken into account. This proves that the growth of needle-crystals is stable with respect to side-branching.
\end{abstract}

\section{Introduction}

During the last decade tremendous efforts have been made by both experimentalists and theoreticians to understand the dynamics of pattern forming systems in such various fields as hydrodynamics, reactions kinetics or aggregation processes. Despite the apparent variety of mechanisms involved in these phenomena some unifying features are now emerging from these works. There remain a lot of open questions and unsolved problems, in particular regarding the mathematical status of the theory, nevertheless a systematic approach to this kind of problem seems nowadays to be more than just a dream. We refer the reader to [L1-2] and [KKL] for comprehensive reviews of these subjects, and to [CE] for an nice introduction to the physics and mathematics of extended systems (i.e., systems that are not confined in some finite volume). In this paper we will be concerned with a specific problem that typically occurs in the study of extended systems: the determination of the continuous part of the excitation spectrum of a stationary (or periodic) state. This is an essential step in the study of such states since it allows to analyze its stability with respect to propagating excitations as opposed to localized modes which correspond to the discrete spectrum. The latter may be much more difficult

* Present address: Department of Mathematics, University of Toronto, Toronto Canada, M5S1A1 
to understand, and will not be discussed here, but in any case it is quite clear that a good control on the continuous spectrum will be a necessary prerequisite to any serious theoretical or numerical analysis. We will concentrate on a particular model, but our technique should also apply to various other situations of interest.

To be specific, we will consider the solidification of a pure substance from its undercooled melt. Neglecting non-equilibrium processes, we may assume that the state of this system is entirely described by the temperature field $T$ and the position of the phase boundary $\Gamma$. The dynamics of this solidification front is controlled by the heat flux which is conventionally given by Fick's law $\mathbf{J} \equiv-K \nabla T$, where $K$ is the thermal conductivity tensor and depends on the phase. As the crystal develops, driven by the undercooling of the melt, the latent heat is released at the interface. The solid phase can only keep growing if the heat flux manages to transport this perturbing energy far away from the front, thus avoiding an excessive increase of the temperature in the neighborhood of the interface. In terms of the specific heat $C$ the local variation of the temperature is given by $C \partial_{t} T=-\nabla \cdot \mathbf{J}$; introducing the thermal diffusion tensor $D \equiv K / C$ we get the equation

$$
\partial_{t} T=\nabla \cdot D \nabla T \text { outside } \Gamma,
$$

for the evolution of $T$. If $T_{M}$ is the equilibrium melting temperature of the substance we have to impose the boundary condition

$$
\left.T\right|_{\Gamma}=T_{M}
$$

Latent heat generation at the phase boundary is easily seen to require the following jump condition on $\Gamma$ :

$$
\left.C \hat{\mathbf{n}} \cdot D \nabla T\right|_{\text {sol }}-\left.C \hat{\mathbf{n}} \cdot D \nabla T\right|_{\text {liq }}=L \hat{\mathbf{n}} \cdot \mathbf{v}
$$

where $L$ is the latent heat, $\mathbf{v}$ the velocity of the front and $\hat{\mathbf{n}}$ its unit normal. Finally we should require the asymptotic undercooling condition,

$$
\lim _{t \rightarrow-\infty} T=T_{0}<T_{M}
$$

For simplicity we will restrict ourself to the two-dimensional situation, although the transposition to three dimensions should not be problematic. The thermal diffusion tensor being a constant scalar in the liquid phase one easily checks that stationary plane interfaces exist provided the undercooling satisfies the very restrictive condition

$$
\Delta \equiv \frac{T_{M}-T_{0}}{L / C}=1
$$

while the front velocity is left arbitrary. A more general class of stationary solutions was obtained by Ivantsov [I] by separation of variables in parabolic coordinates. These so-called needle-crystal solutions are characterized by a parabolic front with tip curvature radius $\rho$, and a constant propagation velocity $v$. These quantities are linked to the undercooling by the relation

$$
\Delta=\sqrt{\pi p} \mathbf{e}^{p} \operatorname{erfc}(\sqrt{p}),
$$

where $p \equiv p v / 2 D$ is called Péclet number. The plane interfaces are clearly obtained in the $\rho \rightarrow \infty$ limit. It is interesting to remark that all these solutions have $T=T_{M}$ 
in the solid phase, so they do not depend on the detailed properties of the crystalline phase, in particular they remain solutions of the symmetric model introduced by Langer and Turski in [LT]. There it was assumed that the thermal diffusion tensor and specific heat of both phases are identical; this greatly simplifies the analytical work with the model without changing too much its physics. We shall therefore also consider this model. In this setting it is not hard to show that there is no other smooth stationary fronts.

Unfortunately two problems arise with Ivantsov's solutions: first they are completely unstable in the sense that if we linearize the evolution equation (1.1-4) around them we find exponential solutions with arbitrary large growth rates (see [LM1]). Thus these stationary states should be dynamically irrelevant for the system. The second point is that at fixed undercooling, (1-6) allows for a continuum of solutions parametrized by say the velocity, in patent disagreement with the experiments which clearly show a reproducible selection of the front velocity. The first step toward the solution of these two problems is to realize that the boundary condition (1.2) is only valid for plane fronts. As soon as the interface becomes curved the equilibrium temperature changes because the attraction of the crystal on the molecules of the liquid is modified, this is the so-called Gibbs-Thomson effect (see [L2] for a more extensive discussion). In the two-dimensional case this effects in changing (1.2) to

$$
\left.T\right|_{\Gamma}=T_{M}-\gamma \kappa,
$$

where $\gamma$, a surface tension parameter, is a material coefficient which may depend on the orientation of the interface with respect to the crystalline axis, and $\kappa$ is the curvature of the front. This new term does clearly not affect the plane solutions, but it makes the separation of variables in parabolic coordinates impossible and thus breaks the Ivantsov solutions. We don't know any explicit stationary solution of this new problem, in fact we don't even know if such a solution exists. However some intuition was gained by the study of simpler local models of interface dynamics: the boundary-layer and the geometric models. There it was found (see [BGLS1-2] and [BKKL1-2] for the seminal papers) that the anisotropy of the surface tension $\gamma$ was a necessary condition for the existence of solutions, furthermore the Ivantsov-like continuum of steady-states that occurred at $\gamma=0$ breaks down into a discrete set. All these modes are linearly unstable except for one which becomes stable at some critical $\gamma_{c}$ thus providing the desired velocity selection. Extensive numerical calculations $[\mathrm{M}, \mathrm{KKL} 1, \mathrm{KL}]$, and convincing (but non-rigorous) mathematical studies [BHL, BL, CCRL, CCMR, BP] have then shown that an analogous scenario is likely to occur in the full non-local model, although some controversies remain on this subject (see in particular [VSW]). We shall not be concerned with these problems here but merely assume the existence of some needle-crystal solution (in Sect. 3 we will show that this must be almost parabolic) and discuss its linear stability.

As mentioned above we must distinguish between two kinds of potential instabilities: modes remaining localized in the frame moving with the non-perturbed stationary front and which arise from the discrete spectrum. They are usually called tip-splitting modes since they would destroy the identity of a single needle. Such modes, which may be important in some other systems, have been argued to be inefficient in the considered operating regime of the crystal. This was done 
mainly on the basis of the analogy with the previously discussed velocity selection mechanism in local models. There is however no definitive evidence for this to be true, and we hope that the results of this work will make possible a precise (numerical) analysis of this eigenvalue problem. The second type of instabilities are related to the continuous spectrum and are thus moving with respect to the stantionary front. In fact the crystals observed thus far in laboratories [GSA, HG1-2] or numerical simultations [SGM1-2] show the emergence of side-branches organized in characteristic dentritic patterns along the sides of the needle crystal. Moreover this structure remains fixed in the laboratory frame, indicating that the side-branching modes should in fact propagate down the needle at a speed approximately opposite to the front velocity. Some numerical computations [LM2-3, EM], albeit not very convincing, support the existence of an unstable component in the continuous spectrum. This would provide, via some (yet unknown) bifurcation mechanism, a good explanation of the dentritic growth of needle crystals. On the other hand it has been advocated by J. S. Langer (see [LP, L3, BBL]) that side-branching could be a purely noise-driven phenomenon and that the needle crystal should thus be a stable attractor. It is the lack of clear-cut argument for or against side-branching instability that ultimately motivated our interest in this work.

In the degenerate case of plane fronts, the stability analysis was first achieved by Mullins and Sekerka [MS1-2], they found a purely continuous spectrum with an unstable band at small wave numbers. For genuine needle-crystals the lack of translation invariance makes the discussion much more complicated and allows, at least in principle, for the existence of discrete spectrum.

In the remainder of this section we explain the heuristic basis of our method, postponing the technical implementation to the subsequent sections. In order to simplify the exposition we will suppose here that the stationary needle-crystal has an exact parabolic shape, an assumption that is not necessary and will later be relaxed. Introducing Cartesian coordinates $x$ and $y$ in the plane with the $y$-axis in the growth direction, the front $\Gamma_{t}$ at time $t$ is described by

$$
\left(\begin{array}{c}
x \\
-x^{2} / 2 \rho+v t
\end{array}\right)+\frac{f(t)}{\sqrt{x^{2}+\rho^{2}}}\left(\begin{array}{l}
x \\
\rho
\end{array}\right),
$$

where $f$ is the normal deviation from the stationary solution. Using the free heat kernel $P^{t} \equiv e^{D \nabla^{2} t}$, the second Green's Identity allows to rewrite (1.1),(1.3) and (1.4) as a single integral equation

$$
T(t)=T_{0}+\frac{L}{C} \int_{-\infty}^{t} d \tau \int_{\Gamma_{\tau}} P^{t-\tau} v^{\perp}(\tau) d s
$$

wher $d s$ is the arclength on the interface. Restricting this relation to the front itself and using Eq. (1.7) we finally get

$$
\frac{\Delta}{p}-\sigma \kappa=\left.\frac{1}{p} \int_{-\infty}^{t} d \tau \int_{\Gamma_{\tau}} P^{t-\tau} v^{\perp}(\tau) d s\right|_{\Gamma_{t}},
$$

where we have used the previously defined undercooling and Péclet parameters, and introduced the so-called capillary length $\sigma \equiv C \gamma / p L$. Equation (1.9) which is the non-linear evolution equation for the front, can now be linearized around the 
stationary solution i.e., at $f=0$. This is an elementary but tedious calculation which, after rescaling length and time to the natural units of $\rho$ and $\rho / v$ and Fourier transforming in time, leads to

$$
\begin{aligned}
& \left(-\frac{\sigma}{1+x^{2}} \partial_{x}^{2}+\frac{\sigma x}{\left(1+x^{2}\right)^{2}} \partial_{x}-\frac{\sigma}{\left(1+x^{2}\right)^{3}}-\frac{1}{\sqrt{\left(1+x^{2}\right)}}\right) f(\omega) \\
& \quad+\Lambda(\omega) f(\omega) \equiv A(\omega) f(\omega)=0,
\end{aligned}
$$

where $\Lambda$ is an integral operator which can be expressed as

$$
\Lambda(\omega) f(\omega, x) \equiv \int_{0}^{\infty} \frac{d \tau}{2 \pi \tau} \int_{-\infty}^{\infty} d y \mathbf{e}^{-p W(\tau, x, y)-i \omega \tau}\left\{p+i \omega-p \frac{x^{2}-y^{2}}{2 \tau}\right\} \sqrt{1+y^{2}} f(\omega, y),
$$

with an exponent $W$ given by

$$
W(t, x, y) \equiv \frac{1}{2 t}\left[(x-y)^{2}+\left(t-\frac{x^{2}-y^{2}}{2}\right)^{2}\right] .
$$

These are nice calculations, however the resulting expressions are so involved that one does not really gain any intuitive understanding of the perturbation equation. The main problem clearly lies in the operator $\Lambda$. In fact this is a common difficulty with integral operators. The knowledge of the integral kernel, which is in principle all we need, does not generally tell us what the operator is really doing. In such situations it is often better to switch to another representation namely pseudo-differential operators $(\Psi$-DO). Roughly speaking these are integral operators which are given in terms of a symbol $a(x, \xi, y)$ by the formula

$$
\mathrm{Op}(a) f(x) \equiv \iint \frac{d \xi d y}{2 \pi} a(x, \xi, y) \mathbf{e}^{i \xi(x-y)} f(y)
$$

A differential operator $P\left(x,-i \partial_{x}\right)$ is of course a special case of such a $\Psi$-DO with symbol $P(x, \xi)$, but this class is much larger, including also inverses and even more complicated functions of differential operators. A look at our formula for $\Lambda$ leads us to guess that it can be written like (1.11) with a symbol $\lambda$ of the form $\lambda(\omega ; x, \xi, y)=b(\omega ;(x+y) / 2, \xi) \sqrt{1+y^{2}}$. The function $b$ can be obtained by Fourier transformation with respect to $r \equiv x-y$ as

$$
b(\omega ; X, \xi)=\int \frac{d r d \tau}{2 \pi} \exp \left\{-i(\omega \tau+\xi r)-p\left[r^{2}+(r X-\tau)^{2}\right] / 2 \tau\right\}\{p+i \omega-p r X / \tau\} .
$$

First evaluating the Gaussian integral in $r$, it is then easy to transform the remaining $\tau$ integration into another Gaussian integral. This allows us to write down a symbol for the operator $A(\omega)$ of $(1.10)$ as

$$
\begin{aligned}
a(\omega ; x, \xi, y)= & \frac{\sigma \xi^{2}}{1+x^{2}}+i \frac{\sigma x \xi}{\left(1+x^{2}\right)^{2}}-\frac{\sigma}{\left(1+x^{2}\right)^{2}}-\frac{1}{\sqrt{1+x^{2}}} \\
& +\sqrt{\frac{1+\mathrm{y}^{2}}{1+X^{2}}} \frac{i\left(\omega+\frac{\xi X}{1+X^{2}}\right)+\frac{p}{1+X^{2}}}{\sqrt{\frac{\xi^{2}}{1+X^{2}}+2 i p\left(\omega+\frac{\xi X}{1+X^{2}}\right)+\frac{p^{2}}{1+X^{2}}}}
\end{aligned}
$$


with $X \equiv(x+y) / 2$. Let us forget for a while that this is a symbol, and look at it as a simple function on phase space $\left\{(x, \xi) \in \mathbb{R}^{2}\right\}$ (think of a Schrödinger operator and its classical Hamiltonian), in particular identify $y$ and $x$. The first natural thing to do is to find a canonical transformation to simplify this function. A good candidate seems to be

$$
\zeta \equiv \frac{\xi}{\sqrt{1+x^{2}}} \Rightarrow s \equiv h(x)=\text { arclength on } \Gamma .
$$

If we denote by $g(s)$ the inverse of $h(x)$ and set $\eta \equiv g / \sqrt{1+g^{2}}$ we easily get the estimate

$$
a=\sigma \zeta^{2}+\frac{i(\omega+\zeta \eta(s))}{\sqrt{\zeta^{2}+2 i p(\omega+\zeta \eta(s))}}+o(1)
$$

uniformly in $\zeta$ as $s \rightarrow \infty$. In particular, since $\eta$ behaves like a smooth signum function, our Hamiltonian $a$ has definite (although distinct) limits $a_{ \pm}(\omega ; \zeta)$ as $s \rightarrow \pm \infty$. Quantizing these functions we get two operators $A_{ \pm}(\omega) \equiv \operatorname{op}\left(a_{ \pm}\right)$which are so simple that we can explicitly calculate their spectra (strictly speaking we can not yet talk about spectrum since we did not introduce any Banach space, however the intuitive meaning should be clear):

$$
\operatorname{spec}\left(A_{ \pm}(\omega)\right)=\left\{a_{ \pm}(\omega, \zeta) \mid \zeta \in \mathbb{R}\right\} .
$$

Since $a_{+}(\omega, \zeta)=a_{-}(\omega,-\zeta)$ the two operators $A_{+}$and $A_{-}$are similar, thus we only need to consider $A_{+}$. The problem is now to relate this to the spectrum of $A(\omega)$. There are basically two steps: first find a unitary operator implementing the above canonical transformation, then decouple the left and right sides of the front to allow the limits $s \rightarrow \pm \infty$ to be taken simultaneously. The first step is a little bit tricky since non-linear symplectic maps are not implementable by unitary transformations, it is however possible to do this job modulo a small error. In our case one can even make this error compact. The second step can be done using any decoupling technique used in Schrödinger operator theory since the dominant contribution to $A$ is the $\sigma \zeta^{2}$ term. The well known result is that decoupling can be achieved at the cost of a relatively trace-class perturbation. To deal with all the errors generated in this process we apply Weyl's theorem on the invariance of the essential spectrum with respect to relatively compact perturbations. We refer to the subsequent sections for more details. Once the continuous spectrum of $A(\omega)$ is known, it is easy to get the dispersion relation by imposing the instability condition $0 \in \operatorname{spec}(A(\omega))$ i.e.,

$$
a_{+}(\omega, \zeta)=0
$$

This can be solved for $\omega$, the result is

$$
\omega(\zeta)=-\zeta+i \sigma^{2} p \zeta^{4}\left(\sqrt{1+\frac{1}{\sigma^{2} p \zeta^{2}}}-1\right),
$$

which should be thought of as a relation between the (complex) frequency $\omega$ and the asymptotic wave number $\zeta$ at $s=+\infty$. The asymptotic group velocity is then 
given by

$$
v_{G} \equiv \frac{\partial \operatorname{Re}(\omega)}{\partial \zeta}=-1,
$$

consistent with the requirement of stationarity in the laboratory frame. A look at Eq. (1.13) soon reveals that $\operatorname{Im}(\omega)$ is non-negative, and zero only at $\zeta=0$ (see Fig. 2 in Sect. 6). Thus we arrive at our main result: needle crystals are marginally stable against wide-branching if the Gibbs-Thomson effect is taken into account.

The remainder of this paper is organized as follows: in Sect. 2 the linearized perturbation equation is derived. In order to simplify the exposition we directly implement the coordinate change (1.12) at this level. The needed a priori properties of stationary needle-crystals are obtained in Sect. 3 by analyzing the asymptotic behavior of the non-linear equation (1.9) far away from the tip. In the next section the main analytic estimates on the integral operator $\Lambda$ are done and its (principal) symbol is computed, this is step one in the above discussion. The spectral analysis of the perturbation equation (1.10) is then carried out in Sect. 5, including step two. The equation is solved in Sect. 6, and the stability of needle-crystals is derived.

\section{The Linear Perturbation Equation}

In appropriate units the symmetric model can be written as a free boundary value problem [L2]. The dimensionless reduced temperature

satisfies the heat equation

$$
u \equiv \frac{T-T_{0}}{p L / C}
$$

$$
\frac{\partial u}{\partial t}=\frac{1}{2 p} \Delta_{x} u
$$

in the two phases. In order to agree with standard notation we use $\Delta$ to denote the undercooling parameter and $\Delta_{x}$ for the Laplacian. We recall that the diffusion constant in (2.1) does not depend on the phase. More realistic models can be defined by relaxing this hypothesis, but our result should not be seriously affected by that point. Local thermodynamic equilibrium at the interface imposes a first boundary condition on the solidification front $\Gamma_{t}$

$$
\left.u\right|_{\Gamma_{t}}=\frac{\Delta}{p}-\sigma \kappa,
$$

where $\Delta$ is the dimensionless undercooling parameter (1.5) measuring the departure of the melt temperature from the melting point. The ratio $\Delta / p$ is the reduced equilibrium temperature of a flat interface. The Gibbs-Thomson effect [L2] modifes this temperature when the front is curved, $\kappa$ is the curvature of the interface and $\sigma$ the capillary length. The last ingradient of the model is the local energy conservation at the interface where the latent heat is released. This leads to another boundary condition

$$
v^{\perp}+\frac{1}{2}\left\{\left.\frac{\partial u}{\partial v}\right|_{\text {liq }}-\left.\frac{\partial u}{\partial v}\right|_{\text {sol }}\right\}=0
$$


relating the normal velocity of the front to the discontinuity of the normal heat flux across the phase boundary. Let us now perturb this problem by adding a small external heat source $\varepsilon Q$ in the right-hand side of (2.1). Using the second Green's Identity we can put the perturbed evolution equation together with its boundary condition (2.3) into a single, physically more transparent distributional equation

$$
\frac{\partial u_{\varepsilon}}{\partial t}=\frac{1}{2 p} \Delta_{x} u_{\varepsilon}+\frac{1}{p} v_{\varepsilon}^{\perp} \delta_{\Gamma_{t}^{\varepsilon}}+\varepsilon Q
$$

where $\delta_{\Gamma}$ is the Dirac distribution concentrated on the curve $\Gamma$, i.e., if $s$ is the arclength on $\Gamma$ then for any test function $\varphi$

$$
\left\langle\delta_{\Gamma}, \varphi\right\rangle \equiv \int_{\Gamma} \varphi d s
$$

Quite naturally we restrict ourself to perturbations $Q(t)$ which were not active forever, without loss of generality we may assume that $Q(t)$ vanishes for $t<0$. Then the usual variation of constant procedure yields for any $t_{0}<0$ the integral equation

$$
u_{\varepsilon}(t)=\mathbf{e}^{-G\left(t-t_{0}\right)} u_{0}\left(t_{0}\right)+\frac{1}{p} \int_{t_{0}}^{t} \mathbf{e}^{-G(t-\tau)} v_{\varepsilon}^{\perp}(\tau) \delta_{\Gamma_{\tau}^{\varepsilon}} d \tau+\varepsilon q(t)
$$

with a perturbing term $q(t) \equiv \int_{0}^{t} \mathbf{e}^{-G(t-\tau)} Q(\tau) d \tau$, and a propagator generated by

$$
G \equiv-\frac{\Delta_{x}}{2 p}
$$

If we now assume that in the remote past the system was entirely liquid (i.e., that $\lim _{t \rightarrow-\infty} u_{0}(t)=0$ ), we get from (2.5) and (2.2) a single integral equation for the front

$$
\frac{\Delta}{p}-\sigma \kappa_{\varepsilon}(t)=\left.\left\{\varepsilon q(t)+\frac{1}{p} \int_{-\infty}^{t} \mathbf{e}^{-G(t-\tau)} v_{\varepsilon}^{\perp}(\tau) \delta_{\Gamma_{t}^{\varepsilon}} d \tau\right\}\right|_{\Gamma_{t}^{c}} .
$$

In the absence of surface tension $(\sigma=0)$ Ivantsov [I] found a continuous family of needle-crystal solutions of the non-perturbed problem parametrized by $p>0$ (see [L2] for a short proof). They describe parabolic fronts of varying curvature and speed in the laboratory frame. In our coordinate system all these have unit tip curvature and propagate at unit velocity. The undercooling is then fixed by the relation

$$
\Delta=\sqrt{\pi p} \mathbf{e}^{p} \operatorname{erfc}(\sqrt{p})
$$

Experimentally a unique solution is selected from this continuum. This selection is currently believed to be an effect of the Gibbs-Thomson term. Unfortunately, no needle-crystal solution is known at $\sigma \neq 0$, there is however strong evidence for the existence of a discrete set of solutions when the surface tension is anisotropic i.e., when $\sigma$ depends non-trivially on the orientation of the interface with respect to the crystalline axes (references were given in Sect. 1). This anisotropy seems to be of fundamental importance for the existence of solutions. Our aim here is to 
study the excitation spectrum of such solutions, merely assuming their existence. It is quite easy to see that any mild anisotropy (i.e., such that $\sigma$ remains bounded from below and above on the interface) can in fact be absorbed in a regular change of coordinate which preserves the structure of our problem. In fact the only effect is a trivial renormalization of the function $g(s)$ near the tip, it is thus safe to ignore anisotropy in the following.

Introducing a Cartesian coordinate system $(x, y)$ in the plane with the $y$-axis in the propagation direction of the crystal we describe the non-perturbed interface $\Gamma^{0}$ in the co-moving frame by

$$
y=-\phi(x) .
$$

We assume $\phi \in C^{2}$ to be even and convex, normalized by $\phi(0)=0$ and $\phi^{\prime \prime}(0)=1$. The algebraic arclength on $\Gamma^{0}$ is given by

$$
s=h(x) \equiv \int_{0}^{x} \sqrt{1+\phi^{\prime 2}(u)} d u .
$$

We denote by $g$ the inverse function of $h$, the arclength parametrization of the front is then

$$
\mathbf{x}_{0}(s)=\left(\begin{array}{c}
g(s) \\
-\phi \circ g(s)
\end{array}\right),
$$

while unit tangent and normal vectors are

$$
\mathbf{t}_{0}=\left(\begin{array}{c}
g^{\prime} \\
-\phi^{\prime} \circ g g^{\prime}
\end{array}\right), \quad \mathbf{n}_{0}=\left(\begin{array}{c}
\phi^{\prime} \circ g g^{\prime} \\
g^{\prime}
\end{array}\right) \text {. }
$$

The perturbed front $\Gamma_{t}^{\varepsilon}$ is given by the normal deformation $f_{\varepsilon}$ as

$$
\mathbf{x}_{\varepsilon}(s, t)=\mathbf{x}_{0}(s)+\mathbf{v}_{0} t+\varepsilon f_{\varepsilon}(s, t) \mathbf{n}_{0}(s) .
$$

We now insert (2.11) in the integral equation (2.7) and linearize in $\varepsilon$. We first consider the left-hand side of Eq. (2.7), the curvature is given by

$$
\kappa_{\varepsilon}=\frac{\mathbf{x}_{\varepsilon}^{\prime \prime} \wedge \mathbf{x}_{\varepsilon}^{\prime}}{\left|\mathbf{x}_{\varepsilon}^{\prime}\right|^{3}}
$$

where' denotes differentiation with respect to $s$. Using the Frenet formulae we get

$$
\begin{aligned}
& \mathbf{x}_{\varepsilon}^{\prime}=\left(1+\varepsilon \kappa_{0} f_{\varepsilon}\right) \mathbf{t}_{0}+\varepsilon f_{\varepsilon}^{\prime} \mathbf{n}_{0}, \\
& \mathbf{x}_{\varepsilon}^{\prime \prime}=\varepsilon\left(\kappa_{0}^{\prime} f_{\varepsilon}+2 \kappa_{0} f_{\varepsilon}^{\prime}\right) \mathbf{t}_{0}+\left(\varepsilon f_{\varepsilon}^{\prime \prime}-\varepsilon \kappa_{0}^{2} f_{\varepsilon}-\kappa_{0}\right) \mathbf{n}_{0},
\end{aligned}
$$

with a non-perturbed curvature given by

$$
\kappa_{0}=\frac{\phi^{\prime \prime} \circ g}{\left(1+\phi^{\prime 2} \circ g\right)^{3 / 2}} .
$$

Thus we readily obtain the linearized curvature

$$
\kappa_{\varepsilon} \sim \kappa_{0}+\varepsilon\left(-\partial_{s}^{2}-\kappa_{0}^{2}\right) f_{0} .
$$

Turning to the right-hand side of (2.7) let us introduce the operator

$$
\Gamma_{t}^{\varepsilon}: \varphi(\mathbf{x}) \mapsto \varphi\left(\mathbf{x}_{\varepsilon}(s, t)\right)
$$


and denote its adjoint (in the $L^{2}$-sense) by $\Gamma_{t}^{\varepsilon^{+}}$. In terms of this we can easily rewrite the integral in the right-hand side of (2.7) as

$$
\frac{1}{p} \int_{0}^{\infty} \Gamma_{t}^{\varepsilon} \mathbf{e}^{-G \tau} \Gamma_{t-\tau}^{\varepsilon^{+}}\left[\mathbf{x}_{\varepsilon}^{\prime} \wedge \dot{\mathbf{x}}_{\varepsilon}\right](t-\tau) d \tau .
$$

The $\varepsilon$-derivative of the restriction operator is seen to be

$$
\left.\partial_{\varepsilon} \Gamma_{t}^{\varepsilon}\right|_{\varepsilon=0}=f_{0} \mathbf{n}_{0} \cdot \Gamma_{t}^{0} \nabla
$$

whereas the volume factor gives

$$
\left.\partial_{\varepsilon}\left[\mathbf{x}_{\varepsilon}^{\prime} \wedge \dot{\mathbf{x}}_{\varepsilon}\right]\right|_{\varepsilon=0}=\dot{f}_{0}-\left(f_{0} \mathbf{v}_{0} \cdot \mathbf{t}_{0}\right)^{\prime} .
$$

Thus the derivative of the integrand in (2.13) consists of the three following terms:

$$
\begin{gathered}
f_{0}(t) \mathbf{n}_{0} \cdot \boldsymbol{\Gamma}_{t}^{0} \nabla \mathbf{e}^{-G \tau} \boldsymbol{\Gamma}_{t-\tau}^{0^{+}} v_{0}^{\perp}=f_{0}(t) \mathbf{n}_{0} \cdot \boldsymbol{\Gamma} \nabla \mathbf{e}^{-H \tau} \boldsymbol{\Gamma}^{+} v_{0}^{+}, \\
-\boldsymbol{\Gamma}_{t}^{0} \mathbf{e}^{-G \tau} \nabla \boldsymbol{\Gamma}_{t-\tau}^{0^{+}} \cdot \mathbf{n}_{0} v_{0}^{\perp} f_{0}(t-\tau)=-\boldsymbol{\Gamma} \mathbf{e}^{-H \tau} \nabla \boldsymbol{\Gamma}^{+} \cdot \mathbf{n}_{0} v_{0}^{\perp} f_{0}(t-\tau), \\
\boldsymbol{\Gamma}_{t}^{0} \mathbf{e}^{-G \tau} \boldsymbol{\Gamma}_{t-\tau}^{0^{+}}\left[\dot{f}_{0}-\left(f_{0} \mathbf{v}_{0} \cdot \mathbf{t}_{0}\right)^{\prime}\right](t-\tau)=\boldsymbol{\Gamma} \mathbf{e}^{-H \tau} \boldsymbol{\Gamma}^{+}\left[\dot{f}_{0}-\left(f_{0} \mathbf{v}_{0} \cdot \mathbf{t}_{0}\right)^{\prime}\right](t-\tau),
\end{gathered}
$$

where $H \equiv G-\mathbf{v}_{0} \cdot \nabla$ and $\boldsymbol{\Gamma}=\Gamma_{0}^{0}$. Since $\partial_{s} \boldsymbol{\Gamma}=\mathbf{t}_{0} \cdot \Gamma \nabla$, we are able to rewrite the integrand as

$$
f_{0}(t) \mathbf{n}_{0} \cdot \boldsymbol{\Gamma} \nabla \mathbf{e}^{-\boldsymbol{H} \tau} \boldsymbol{\Gamma}^{+} v_{0}^{\perp}+\boldsymbol{\Gamma} \mathbf{e}^{-H \tau}\left(\partial_{t}-\mathbf{v}_{0} \cdot \nabla\right) \boldsymbol{\Gamma}^{+} f_{0}(t-\tau) .
$$

The first term in this expression gives rise to a multiplication operator, the corresponding integral is easily identified to be

$$
\begin{aligned}
\frac{1}{p} \int_{0}^{\infty} d \tau\left[\mathbf{n}_{0} \cdot \Gamma \nabla \mathbf{e}^{-H \tau} \Gamma^{+} v_{0}^{\perp}\right](s) \\
=-p \int_{0}^{\infty} \frac{d \tau}{2 \pi \tau^{2}} \int_{-\infty}^{\infty} d s^{\prime} \mathbf{n}_{0}(s) \cdot\left(\mathbf{x}_{0}(s)-\mathbf{x}_{0}\left(s^{\prime}\right)\right. \\
\left.\quad+\mathbf{v}_{0} \tau\right) \mathbf{v}_{0} \cdot \mathbf{n}_{0}\left(s^{\prime}\right) \mathbf{e}^{-p\left|\mathbf{x}_{0}(s)-\mathbf{x}_{0}\left(s^{\prime}\right)+\mathbf{v}_{0} \tau\right|^{2} / 2 \tau} \\
=\frac{1}{2}\left\{\left.\frac{\partial u_{0}}{\partial v}\right|_{\text {liq }}+\left.\frac{\partial u_{0}}{\partial v}\right|_{\text {sol }}\right\} \equiv j_{0}^{\perp}(s),
\end{aligned}
$$

that is the non-perturbed heat flux through the interface (we will however not use this identification). We finally get the linearized equation for the dynamics of the normal deformation $f$ driven by the external perturbation $q$ as

$$
A f \equiv\left(-\sigma \partial_{s}^{2}-\sigma \kappa_{0}^{2}-j_{0}^{\perp}+\Lambda\right) f=q
$$

where the operator $\Lambda$ is given by

$$
\Lambda f(t)=\frac{1}{p} \int_{0}^{\infty} d \tau \Gamma \mathbf{e}^{-H \tau}\left(\partial_{t}-v_{0} \cdot \nabla\right) \Gamma^{+} f(t-\tau) .
$$

Consideration of causality forces us to restrict ourself to solutions $f$ that vanish for negative times. Since on the other hand it is quite natural to assume that $f$ is exponentially bounded in the limit $t \rightarrow \infty$ if $q$ is, the Fourier transform $\hat{f}(\omega) \equiv \int_{0}^{\infty} f(t) \mathbf{e}^{-i \omega t} d t$ should be analytic in some half-plane $\mathbb{C}_{c} \equiv\{\omega \mid \operatorname{Im} \omega<c\}$. 
Introducing the operator

$$
\hat{\Lambda}(\omega) \equiv \frac{1}{p} \int_{0}^{\infty} \boldsymbol{\Gamma} \mathbf{e}^{-(H+i \omega) \tau}\left(i \omega-\mathbf{v}_{0} \cdot \nabla\right) \Gamma^{+} d \tau,
$$

we can reduce the linearized equation to

$$
\hat{A}(\omega) \hat{f}(\omega) \equiv\left(-\sigma \partial_{s}^{2}-\sigma \kappa_{0}^{2}-j_{0}^{\perp}+\hat{\Lambda}(\omega)\right) \hat{f}(\omega)=\hat{q}(\omega) .
$$

Remark that by our judicious choice of parametrization of the free interface the differential part in the operator $\hat{A}$ has been made uniformly elliptic (compare with 1.10), reflecting the dissipative nature of the problem. This is an important advantage over earlier treatments of the question since we will see that $-\sigma \partial_{s}^{2}$ is the dominant term in Eq. (2.17).

\section{A Priori Properties of Stationary Needle-Crystals}

In this section we derive some a priori properties of stationary solutions of the front equation (2.7). We suppose, as in Sect. 2, that $\phi$ is an even, smooth (i.e., $C^{2}$ ) and convex function, normalized by the conditions $\phi(0)=0$ and $\phi^{\prime \prime}(0)=1$. We also assume that $\kappa_{0} \rightarrow 0$ at infinity thus excluding oscillations of the front temperature there.

Theorem 3.1. Any stationary solution $\Gamma_{t}=\{y=t-\phi(x)\}$ of the front equation corresponding to an undercooling $0<\Delta<1$ and satisfying the above assumptions is parabolic at infinity in the sense that for some constants $C_{1}$ and $C_{2}$ we have

$$
0<C_{1} \leqq \frac{\phi(x)}{x^{2}} \leqq C_{2}<\infty
$$

for all $x$. Assuming further that the second derivative $\phi^{\prime \prime}$ is bounded, the heat flux across the interface vanishes at infinity, i.e.,

$$
\lim _{s \rightarrow \pm \infty} j_{0}^{\perp}(s)=0 .
$$

Remark. A more careful analysis shows that in fact $j_{0}^{\perp}(s)=O\left(s^{-1 / 2}\right)$ at infinity. This slow decay at infinity should have important consequences on the structure of the discrete spectrum.

Let us proceed to the proof. It follows from Eq. (2.7) that

$$
\frac{\Delta}{p}=\lim _{x \rightarrow \infty} \int_{0}^{\infty} \frac{d \tau}{\tau} \int_{-\infty}^{\infty} d y \mathbf{e}^{-p\left\{(y-x)^{2}+(\phi(y)-\phi(x)+\tau)^{2}\right\} / 2 \tau},
$$

where $d \tau \equiv d \tau / 2 \pi$. Now by a change of variable we can put the last integral into the quasi-Gaussian form

$$
I(x)=\int_{-\infty}^{\infty} \int_{-\infty}^{\infty} d \sigma d \tau \mathbf{e}^{-(p / 2) Q(x, x+\sigma)[\sigma, \tau]}
$$

where the quadratic form $Q$ is given by the matrix

$$
Q(x, y)=\left(\begin{array}{cc}
1+G(x, y)^{2} & G(x, y) \\
G(x, y) & 1
\end{array}\right) \text { with } G(x, y)=\frac{\phi(x)-\phi(y)}{x-y} .
$$


From the convexity of $\phi$ one easily infers that the function $G$ is monotone increasing in both arguments. As a consequence the following limits exist and coincide:

$$
\pm A \equiv \lim _{x \rightarrow \pm \infty} \frac{\phi(x)}{x}=\lim _{x \rightarrow \pm \infty} \phi^{\prime}(x)=\lim _{x \rightarrow \pm \infty} G(x, y)=\lim _{x \rightarrow \pm \infty} G(x, x+y)
$$

Correspondingly the asymptotic normal velocity of the front

$$
\lim _{s \rightarrow \pm \infty} v_{0}^{\perp}(s)=\left(1+A^{2}\right)^{-1 / 2}
$$

also exists.

We consider first the case of non-vanishing asymptotic normal velocity. Then the quadratic form (3.3) has a proper limit as $x \rightarrow \infty$ which is independent of its second argument and Lebesgue's dominated convergence theorem shows that the limit of the integral (3.2) is indeed a Gaussian integral. Its evaluation gives, by (3.1), the result $\Delta=1$ which generalizes the well known fact that a stationary plane interface can only exist at unit undercooling [L2].

In cases where the asymptotic normal velocity vanishes (i.e., $A=\infty$ ) the situation is more subtle since the quadratic form $Q$ has no limit as $x \rightarrow \infty$. Nevertheless for $\sigma \tau \notin]-2 x, 0$ [ we can use the estimate (we only consider $x \rightarrow \infty$, the opposite case is completely similar)

$$
Q(x, x+\sigma \tau)[\sigma, \tau] \geqq\left(1+G(x, x+\sigma \tau)^{2}\right) \sigma^{2}+\tau^{2},
$$

from which we get by Lebesgue's theorem

$$
\lim _{x \rightarrow \infty} \int_{\sigma \tau \notin]-2 x, 0[} d \sigma d \tau \mathbf{e}^{-(p / 2) Q(x, x+\sigma \tau)[\sigma, \tau]}=0 .
$$

Let us denote by $\sim$ the equivalence modulo $o(1)$ quantities as $x \rightarrow \infty$, then making the previous change of integration variable backward and using a well known integral representation of modified Bessel function (see [ER]) we obtain the new expression

$$
\begin{aligned}
I(x) & \sim \int_{0}^{\infty} \frac{d \tau}{\tau} \int_{-x}^{x} d y \mathbf{e}^{-p\left\{(x-y)^{2}+(\phi(x)-\phi(y)-\tau)^{2}\right\} / 2 \tau} \\
& \sim \frac{1}{\pi} \int_{-x}^{x} d y K_{0}(p d(x, y)) \mathrm{e}^{p(\phi(x)-\phi(y))}
\end{aligned}
$$

where we have set $d(x, y)=\sqrt{(x-y)^{2}+(\phi(x)-\phi(y))^{2}}$. Let us introduce the auxiliary function

$$
\varphi(z) \equiv \frac{\mathbf{e}^{z}}{\pi} K_{0}(z)
$$

which is analytic except for a logarithmic singularity at the origin. The relevant behavior of $\varphi$ is given by (see [AS])

$$
\begin{aligned}
& \varphi(z)=-\frac{1}{\pi} \log z+\mathbf{O}(1) \quad \text { as } \quad z \rightarrow 0 \\
& \varphi(z)=\frac{1}{\sqrt{2 \pi z}}\left(1+\mathbf{O}\left(z^{-1}\right)\right) \quad \text { as } \quad z \rightarrow+\infty
\end{aligned}
$$


In terms of this function and of $S(x, y)=d(x, y)-(\phi(x)-\phi(y))$ we can rewrite

$$
I(x) \sim I_{\varphi}(x) \equiv \int_{-x}^{x} d y \varphi(p d(x, y)) \mathrm{e}^{-p S(x, y)} .
$$

To handle this integral we first prove the

Lemma 3.2. An integrable singularity of $f$ at the origin does not contribute to $I_{f}(x)$ as $x \rightarrow \infty$ in the sense that for such $f$ we have

$$
I_{f}(x) \sim \int_{\substack{-x<y<x \\ p d(x, y)>\rho}} d y f(p d(x, y)) \mathbf{e}^{-p S(x, y)}
$$

for any positive $\rho$.

Proof. Let $I_{0}$ be the integral (3.6) but on the set $\{y \in[-x, x] \mid p d(x, y)<\rho\}$. We have to show that $I_{0}(x) \sim 0$. Since one easily checks that the function $y \mapsto d(x, y)$ is monotone decreasing on the interval $[0, x]$, the change of integration variable $t=p d(x, y)$ allows us to write for large $x$

$$
\left|I_{0}(x)\right| \leqq \frac{1}{p} \int_{0}^{\rho} d t \frac{|f(t)|}{J(x, t)}
$$

with the Jacobian

$$
J(x, t)=-\partial_{y} d(x, y)=\frac{1+\phi^{\prime}(y) G(x, y)}{\sqrt{1+G(x, y)^{2}}} .
$$

But the monotony of $G$ implies

$$
G(x, y) \geqq G(y, y)=\phi^{\prime}(y) \geqq \phi^{\prime}\left(x-\frac{\rho}{p}\right),
$$

thus we conclude that for large $x$,

$$
J(x, t) \geqq \frac{1}{2} \phi^{\prime}\left(x-\frac{\rho}{p}\right) \rightarrow \infty \quad \text { uniformly in } t \text { as } x \rightarrow \infty,
$$

from which the result follows.

Thus by the first estimate in (3.4) we may replace $\varphi(z)$ by $(2 \pi z)^{-1 / 2}$ in any neighborhood of zero in the integral (3.5) without changing the limiting behavior of $I_{\varphi}$. To control the contribution of infinity we need the

Lemma 3.3. Assume that for some positive $\varepsilon$ the function $f$ satisfies $f(z)=O\left(z^{-1-\varepsilon}\right)$ at infinity, then we have

for any positive $\rho$.

$$
I_{f}(x) \sim \int_{\substack{-x<y<x \\ p d(x, y)<\rho}} d y f(p d(x, y)) \mathbf{e}^{-p S(x, y)}
$$

We skip the very simple proof. By the second estimate in (3.4) we may also replace $\varphi(z)$ by $(2 \pi z)^{-1 / 2}$ in any neighborhood of infinity in Integral (3.5), thus finally

$$
I(x) \sim \int_{-x}^{x} d y \frac{\mathbf{e}^{-p S(x, y)}}{\sqrt{2 \pi p d(x, y)}}
$$


We now consider two cases, let us first assume that $\limsup _{x \rightarrow \infty} \frac{\phi(x)}{x^{2}}=\infty$. Since for $0 \leqq y \leqq x$ one easily shows that

$$
d(x,-y) \geqq d(x, y) \geqq(x-y) \frac{\phi(x)}{x},
$$

we can write

$$
\begin{aligned}
I(x) & \leqq 2 \int_{0}^{x} \frac{d y}{\sqrt{2 \pi p d(x, y)}}+o(1) \\
& \leqq 2 \sqrt{\frac{x}{\phi(x)}} \int_{0}^{x} \frac{d y}{\sqrt{2 \pi p(x-y)}}+o(1) \\
& \leqq 2 \sqrt{\frac{2}{p \pi}} \sqrt{\frac{x^{2}}{\phi(x)}}+o(1)
\end{aligned}
$$

which vanishes as $\frac{\phi(x)}{x^{2}}$ tends to infinity. Thus in this case we must have $\Delta=0$.

If we now assume that $\liminf _{x \rightarrow \infty} \frac{\phi^{\prime}(x)}{x}=0$, it then follows that $\liminf _{x \rightarrow \infty} \frac{\phi(x)}{x^{2}}=0$. The function $y \mapsto S(x, y)$ is readily seen to be monotone decreasing on $[-x, x]$, thus for $0 \leqq y \leqq x$ we have for some positive constant $C$

$$
S(x,-y) \geqq S(x, 0)=\frac{x^{2}}{\phi(x)+\sqrt{x^{2}+\phi(x)^{2}}} \geqq C \frac{x^{2}}{\phi(x)} .
$$

Splitting the integral (3.7) in two parts $I_{ \pm}$according to the sign of $y$, the first piece is controlled by

$$
\begin{aligned}
I_{-}(x) & \leqq \sqrt{\frac{x}{\phi(x)}} \int_{0}^{x} d y \frac{\mathbf{e}^{-C x^{2} / \phi(x)}}{\sqrt{2 \pi p(x-y)}} \\
& \leqq \sqrt{\frac{2}{p \pi}} \sqrt{\frac{x^{2}}{\phi(x)}} \mathbf{e}^{-C x^{2} / \phi(x)}
\end{aligned}
$$

which vanishes as $\frac{\phi(x)}{x^{2}} \rightarrow 0$. In the second piece we set $y=\left(1-t^{2}\right) x$, then

$$
\left.\begin{array}{l}
S\left(x,\left(1-t^{2}\right) x\right)=\frac{x t^{2}}{\sqrt{1+G^{2}}+G} \\
d\left(x,\left(1-t^{2}\right) x\right)=x t^{2} \sqrt{1+G^{2}}
\end{array}\right\} \text { where } G \equiv G\left(\left(1-t^{2}\right) x, x\right)
$$

and a short calculation gives the expression

$$
I_{+}(x)=\frac{1}{p} \sqrt{\frac{2}{\pi}} \int_{0}^{1} d t \sqrt{\frac{p x}{\sqrt{1+G^{2}}}} \exp \left(-t^{2}\left\{\frac{p x}{\sqrt{1+G^{2}}+G}\right\}\right)
$$


Now since $G \leqq \phi^{\prime}(x)$, the quantity $x / G$ diverges as $\phi^{\prime}(x) / x$ vanishes, but then the last integral is easily seen to converge to $1 / p$. Thus in this case we conclude that $\Delta=1$.

Summing up we have seen that, for values of $\Delta$ corresponding to non-degenerate Ivantsov solutions (i.e., for $p>0$ in (2.8)), the stationary fronts are almost parabolic in the sense that

$$
0<\liminf _{x \rightarrow \infty} \frac{\phi^{\prime}(x)}{x} \leqq \liminf _{x \rightarrow \infty} \frac{\phi(x)}{x^{2}} \leqq \limsup _{x \rightarrow \infty} \frac{\phi(x)}{x^{2}}<\infty
$$

which clearly implies the first assertion of the theorem. It is then very natural to make the further assumption that $\phi^{\prime \prime}$ is bounded. Under this hypothesis let us now discuss the behavior of the heat flux across the front at infinity. We begin with a simple

Lemma 3.4. The function $\chi(X, r) \equiv G\left(X+\frac{r}{2}, X-\frac{r}{2}\right)$ satisfies the estimate

uniformly in $r$.

$$
0<C_{1} \leqq \frac{|\chi(X, r)|}{|X|} \leqq C_{2}<\infty
$$

Proof. Remark that $\chi$ is odd in its first and even in its second argument, thus we may take $X, r>0$. Assume first $|X|>\frac{r}{2}$. Since by (3.8) there exists a constant $C_{1}$ such that $\phi^{\prime}(x) \geqq C_{1} x$ for all positive $x$, we have in fact the lower bound

$$
\chi(X, r) \geqq \frac{C_{1}}{2} \int_{-1}^{1}\left(X+t \frac{r}{2}\right) d t=C_{1} X
$$

The upper bound follows from the boundedness of $\phi^{\prime \prime}$ in exactly the same way. On the other hand if $|X|<\frac{r}{2}$ the parity of $\phi$ gives to the identity

$$
\chi(X, r)=\frac{2 X}{r} \chi\left(\frac{r}{2}, 2 X\right),
$$

which leads us back to the first case.

From (2.14) we get, after some elementary manipulations

$$
j_{0}^{\perp} \circ h(x) \sim \int_{0}^{\infty} \frac{p d \tau}{\tau^{2}} \int_{-\infty}^{\infty} d y \frac{(x-y) \phi^{\prime}(x)-(\phi(x)-\phi(y))}{\sqrt{1+\phi^{\prime}(x)^{2}}} \mathbf{e}^{-p\left\{(x-y)^{2}+(\phi(x)-\phi(y)-\tau)^{2}\right\} / 2 \tau} .
$$

Taylor's formula applied to the function $\phi$ gives the uniform bound

$$
\left|(x-y) \phi^{\prime}(x)-(\phi(x)-\phi(y))\right| \leqq C(x-y)^{2},
$$

from which we obtain, in the same way as before, the following quasi-Gaussian bound on the absolute value $J(x)$ of the integral in (3.9),

$$
J(x) \approx \frac{1}{x} \int_{-\infty}^{\infty} \int_{-\infty}^{\infty} d \sigma d \tau \sigma^{2} \mathbf{e}^{-(p / 2) Q(x, x+\sigma \tau)[\sigma, \tau]}
$$


Here the notation $f(x) \approx g(x)$ means that $f(x)=O(g(x))$ as $x$ tends to infinity. As before, we may restrict integration to the domain where $-2 x<\sigma \tau<0$ and, after some calculations, get the asymptotic formula (see [Er])

$$
\begin{aligned}
J(x) & \approx \frac{1}{\pi x} \int_{-x}^{x} d y K_{1}\left((d(x, y)) \frac{(x-y)^{2}}{d(x, y)} \mathbf{e}^{p(\phi(x)-\phi(y))}\right. \\
& \approx \frac{1}{x} \int_{-x}^{x} d y(x-y)^{2} \psi(p d(x, y)) \mathbf{e}^{-p S(x, y)}
\end{aligned}
$$

where we have introduced the new function

$$
\psi(z) \equiv \frac{\mathbf{e}^{z}}{\pi z} K_{1}(z)
$$

This function behaves like (see [AS] for Bessel's functions)

$$
\begin{array}{ll}
\psi(z)=\frac{1}{\pi z^{2}}+O(\log (z)) & \text { as } \quad z \rightarrow 0, \\
\psi(z)=\frac{1}{\sqrt{2 \pi z^{3}}}\left(1+O\left(z^{-1}\right)\right) & \text { as } \quad z \rightarrow+\infty
\end{array}
$$

Using the Lemma 3.2 with the above estimates we can discard the singularity at $x=y$ and rewrite

$$
J(x) \approx \int_{-x}^{x} d y \frac{(x-y)^{2}}{x d(x, y)^{3 / 2}}
$$

But by Lemma 3.4

$$
\begin{aligned}
d(x, y) & =(x-y) \sqrt{1+G(x, y)^{2}} \\
& \geqq C \sqrt{1+(x+y)^{2}}(x-y) .
\end{aligned}
$$

Insertion of the estimates (3.12) in (3.11) finally gives $J(x) \sim 0$.

\section{A Principal Symbol for the Integral Operator $\hat{\Lambda}(\omega)$}

In this section we focus on the integral operator given by (2.16) and which can be written more explicitly as

$$
\begin{aligned}
\hat{\Lambda}(\omega) f(s)= & \int_{0}^{\infty} \frac{d \tau}{\tau} \mathbf{e}^{-(p+2 i \omega) \tau / 2} \int_{-\infty}^{\infty} d s^{\prime} \mathbf{e}^{-p d^{2}\left(g(s), g\left(s^{\prime}\right)\right) / 2 \tau+p\left(\phi \circ g(s)-\phi \circ g\left(s^{\prime}\right)\right)} \\
& \cdot\left\{p+i \omega-\frac{p}{\tau}\left(\phi \circ g(s)-\phi \circ g\left(s^{\prime}\right)\right)\right\} f\left(s^{\prime}\right) .
\end{aligned}
$$

Applying the Cauchy theorem to deform the integration contour we get the more 
symmetric expression

$$
\begin{aligned}
\hat{\Lambda}(\omega) f(s)= & p \int_{0}^{\infty} \frac{d \tau}{\tau} \mathbf{e}^{-p(1+\delta) \tau / 2} \int_{-\infty}^{\infty} d s^{\prime} \mathbf{e}^{-p(1+\delta) d^{2}\left(g(s), g\left(s^{\prime}\right)\right) / 2 \tau+p\left(\phi \circ g(s)-\phi \circ g\left(s^{\prime}\right)\right)} \\
& \cdot\left\{1+i v-\frac{1+\delta}{\tau}\left(\phi \circ g(s)-\phi \circ g\left(s^{\prime}\right)\right)\right\} f\left(s^{\prime}\right),
\end{aligned}
$$

where we introduced the notation

$$
v \equiv \frac{\omega}{p}, \quad \delta \equiv \sqrt{1+2 i v}-1
$$

Our goal is to compute a principal symbol for this operator. Let us briefly explain what we mean by that, intentionally avoiding all kinds of technicalities. A function $p(x, \xi, y)$ is a symbol for the operator $P$ acting on some decent subspace of $L^{2}(\mathbb{R})$ (typically the Schwartz space $\mathscr{S}$ ) if

$$
P f(x)=\mathrm{Op}(p) f(x) \equiv \int \mathrm{e}^{i(x-y) \xi} p(x, \xi, y) f(y) d \xi d y .
$$

Clearly the symbol of an operator is not uniquely determined. To remove this indeterminacy we must, loosely speaking, fix the order in which the operators $x$ and $\partial_{x}$ act. For example symbols independent of $y$ will be called left symbols, those independent of $x$ right symbols. In the middle lie the symmetric Weyl symbols, of the form $p\left(\frac{x+y}{2}, \xi\right)$, especially interesting because they give rise to self-adjoint operators when they are real. Fourier transform connects this different kind of symbols together, for example one easily checks that the left symbol is given in term of the right one by the formula

$$
p_{l}(x, \xi)=\int \mathbf{e}^{-i(x-y)(\xi-\eta)} p_{r}(\eta, x-y) d \eta d y .
$$

We will say that a symbol $p$ is a principal symbol for $P$ if the operator $P-O p(p)$ has an extension to $L^{2}(\mathbb{R})$ which is compact relative to $P$. Thus even in a fixed class of symbols, say Weyl symbols, the principal symbol is only determined up to the Weyl symbol of a (relatively) compact operator. The main reason to deal with symbols rather than with kernels is that the former have much nicer properties. Whereas the kernel of an operator product $C=A B$, for example, is given by a quite uninspiring integral, its symbol is almost the product of the symbols of $A$ and $B$. However to really get such a trivial algebra we must restrict ourselves to principal symbols at the cost, of course, of some fine information on the operators. The point is that in many applications one is not interested in such details, then the coarse description given by a principal symbol is largely sufficient. Such a situation arises for example in the determination of dispersion relations for the propagation of waves (or more general excitations) through inhomogeneous extended systems. Then only the essential spectrum is relevant since discrete eigenvalues generally describe localized, non-propagating modes. The main tool in such situations is the stability of the essential spectrum under relatively compact perturbations (i.e., Weyl's principle) which gives great power to the simple calculus of principal symbols. This is exactly the situation we are interested in. 
With this in mind let us now turn to the operator (4.1). We first show that its kernel is essentially supported on the diagonal in the sense of the following:

Lemma 4.1. Let $D$ be an almost conical neighborhood of the diagonal given by

$$
D \equiv\left\{\left(s, s^{\prime}\right)|| g(s)-g\left(s^{\prime}\right) \mid<\sqrt{1+\left|\frac{g(s)+g\left(s^{\prime}\right)}{2}\right|^{2}}\right\}
$$

and define the complex region

$$
\Omega \equiv\{\omega \in \mathbb{C} \mid \operatorname{Re}(\delta)>0\} .
$$

Then under the assumptions of Theorem 3.1 (inclusive the boundedness of $\phi^{\prime \prime}$ ), the integral operator obtained by restricting the kernel of $\hat{\Lambda}(\omega)$ to the complement of $D$ is an analytic function from $\Omega$ to the Hilbert-Schmidt operators on $L^{2}(\mathbb{R})$. Moreover its Hilbert-Schmidt norm satisfies

$$
\|\hat{\Lambda}(\omega)\|_{L^{2}\left(\mathbb{R}^{2} \backslash D\right)}=\mathbf{o}\left(\mathbf{e}^{-p \operatorname{Re} \delta / 2}\right)
$$

as $\operatorname{Re}(\delta)$ tends to infinity.

Remarks.

(i) We shall not distinguish between an integral operator and its integral kernel, whereas the above notation for the Hilbert-Schmidt norm as an $L^{2}$ norm.

(ii) The parameter $\operatorname{Re} \delta$ really measures the distance to the boundary of the analyticity domain $\Omega$. In fact one easily proves the inequality

$$
\operatorname{dist}(\omega, \partial \Omega) \geqq \frac{p}{2} \operatorname{Re} \delta(\operatorname{Re} \delta+2)
$$

which is saturated for imaginary $\omega$.

Proof. The kernel of $\hat{\Lambda}$ can be exactly computed and, up to a constant factor, it is given by $L\left(g(s), g\left(s^{\prime}\right)\right)$ with

$$
L(x, y) \equiv\left\{(1+i v) K_{0}(p(1+\delta) d)+(1+\delta)\left(\frac{S}{d}-1\right) K_{1}(p(1+\delta) d)\right\} \mathbf{e}^{p(\phi(x)-\phi(y))},
$$

where $d=d(x, y)$ and $S=S(x, y)$ are as in the previous section. The $K$ are modified Bessel functions. Let us introduce the new variables $X=(x+y) / 2$ and $r=(x-y)$, then to $D$ corresponds the set

$$
\tilde{D} \equiv\left\{(x, y)|| r \mid<\sqrt{1+X^{2}}\right\} .
$$

Since on the complement of $\tilde{D}$ the distance $d$ is bounded below, the estimates (3.4) and (3.10) allow us to write

$$
L(x, y)=\sqrt{\frac{\pi}{2 p(1+\delta) d}}\left\{(1+\delta) \frac{S}{d}+(i v-\delta)+O\left(\frac{1}{d}\right)\right\} \mathrm{e}^{-p(S+\delta d)} .
$$

As long as $\operatorname{Re}(\delta)>0$ some elementary inequalities further lead to

$$
L(x, y)=\mathbf{e}^{-p \delta d}\left[1+O\left(\frac{\delta-i v}{\operatorname{Re}(\delta)}\right)\right] O\left(d^{-3 / 2}\right) .
$$


On the other hand the Hilbert-Schmidt norm (squared) of the kernel of $\hat{\Lambda}$ on the complement of $D$ is easily seen to be bounded by

$$
N^{2} \equiv \int_{\mathbb{R}^{2} \backslash \tilde{\boldsymbol{D}}}\left|L\left(X+\frac{r}{2}, X-\frac{r}{2}\right)\right|^{2} r^{2} d X d r
$$

Note the last factor in the integrand which comes from the change of variables. By Lemma 3.4 we have

$$
d(x, y)=|r| \sqrt{1+\chi^{2}} \geqq C|r| \sqrt{1+\mathrm{X}^{2}} .
$$

Once (4.4) is inserted into (4.2) it becomes easy to get the integral $N^{2}$ under control. A similar argument for $\partial_{v} L(x, y)$ completes the proof.

To understand the operator $\hat{\Lambda}(\omega)$ near the diagonal (i.e., on $D$ ) we need good approximations of the functions $\phi^{\circ} g(s)-\phi^{\circ} g\left(s^{\prime}\right)$ and $d^{2}\left(g(s), g\left(s^{\prime}\right)\right)$. This is achieved in the two following lemmas under the assumptions of Theorem 3.1.

Lemma 4.2. Let $\eta \equiv \frac{\phi^{\prime}}{\sqrt{1+\phi^{\prime 2}}}$. There is a constant $C$ such that

$$
\left|\phi \circ g(s)-\phi \circ g\left(s^{\prime}\right)-\left(s-s^{\prime}\right) \eta \circ g(s)\right| \leqq C \frac{\left(g(s)-g\left(s^{\prime}\right)\right)^{2}}{1+g(s)^{2}} \leqq C
$$

for any $\left(s, s^{\prime}\right) \in D$.

Proof. We must show that on $\tilde{D}$

$$
\begin{aligned}
& \left|\left(\phi\left(X+\frac{r}{2}\right)-\phi\left(X-\frac{r}{2}\right)\right)-\left(h\left(X+\frac{r}{2}\right)-h\left(X-\frac{r}{2}\right)\right) \eta\left(X+\frac{r}{2}\right)\right| \\
& \leqq C \frac{r^{2}}{1+\left(X+\frac{r}{2}\right)^{2}}
\end{aligned}
$$

Applying Taylor's formula we first obtain

$$
\eta\left(X+\frac{r}{2}\right)-\eta(X)=\frac{r}{2} \int_{0}^{1} \frac{\phi^{\prime \prime}\left(X+t \frac{r}{2}\right)}{\left(1+\phi^{\prime 2}\left(X+t \frac{r}{2}\right)\right)^{3 / 2}} d t
$$

and since on $\tilde{D}$ for large values of $|X|$ we clearly have $\left|X+\frac{r}{2}\right|>C|X|$, we then get

$$
\left|\eta\left(X+\frac{r}{2}\right)-\eta(X)\right| \leqq C \frac{|r|}{1+|X|^{3}} \text {. }
$$


On the other hand an integration by part shows that

$$
\begin{aligned}
F(X, r) & \equiv\left(\phi\left(X+\frac{r}{2}\right)-\phi\left(X-\frac{r}{2}\right)\right)-\left(h\left(X+\frac{r}{2}\right)-h\left(X-\frac{r}{2}\right)\right) \eta(X) \\
& =\frac{r^{2}}{4} \int_{-1}^{1}(\operatorname{sign}(t)-t) \phi^{\prime \prime}\left(X+t \frac{r}{2}\right)\left\{1-\eta(X) \eta\left(X+t \frac{r}{2}\right)\right\} d t,
\end{aligned}
$$

but since on $\tilde{D}$ we have the bound

$$
\left|1-\eta(X) \eta\left(X+\frac{r}{2}\right)\right| \leqq\left(1+\phi^{\prime}(X)\right)^{-1 / 2}\left(1+\phi^{\prime}\left(X+\frac{r}{2}\right)\right)^{-1 / 2} \leqq \frac{C}{1+X^{2}},
$$

we get in fact that

$$
|F(X, r)| \leqq C \frac{r^{2}}{1+X^{2}}
$$

The two inequalities (4.7) and (4.8) together with the simple bound

$$
\left|h\left(X+\frac{r}{2}\right)-h\left(X-\frac{r}{2}\right)\right| \leqq C|r|(1+|X|)
$$

imply the desired estimate (4.5).

Lemma 4.3. There is a constant $C>0$ such that

$$
0 \leqq\left(s-s^{\prime}\right)^{2}-d^{2}\left(\left(g(s), g\left(s^{\prime}\right)\right) \leqq C\left(g(s)-g\left(s^{\prime}\right)\right)^{4}\right.
$$

for any $\left(s, s^{\prime}\right) \in D$.

Proof. The first inequality is clear from the geometric interpretation of the variable $s$ (recall the definitions of the functions $g$ and $h$ in (2.10)). With the new variables $X=\frac{g(s)+g\left(s^{\prime}\right)}{2}$ and $r=\left(g(s)-g\left(s^{\prime}\right)\right)$, the second assertion of the lemma reads

$$
\varepsilon=\left(\frac{h\left(X+\frac{r}{2}\right)-h\left(X-\frac{r}{2}\right)}{r}\right)^{2}-\left[1+\left(\frac{\phi\left(X+\frac{r}{2}\right)-\phi\left(X-\frac{r}{2}\right)}{r}\right)^{2}\right] \leqq C r^{2} .
$$

To verify this let us write $\varepsilon=A+2 B$ with

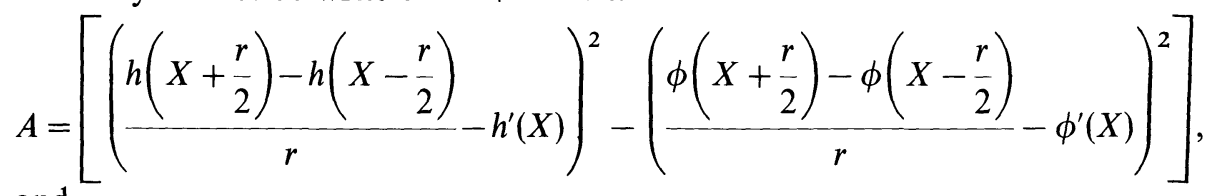

and

$$
\begin{aligned}
B= & {\left[h^{\prime}(X)\left(\frac{h\left(X+\frac{r}{2}\right)-h\left(X-\frac{r}{2}\right)}{r}-h^{\prime}(X)\right)\right.} \\
& \left.-\phi^{\prime}(X)\left(\frac{\phi\left(X+\frac{r}{2}\right)-\phi\left(X-\frac{r}{2}\right)}{r}-\phi^{\prime}(X)\right)\right] .
\end{aligned}
$$


Then the integral formula

$$
\frac{f\left(X+\frac{r}{2}\right)-f\left(X-\frac{r}{2}\right)}{r}-f^{\prime}(X)=\frac{r}{4} \int_{-1}^{1} f^{\prime \prime}\left(X+t \frac{r}{2}\right)(\operatorname{sign}(t)-t) d t
$$

produces the estimate

$$
|A| \leqq C r^{2}
$$

uniformly in $X$. On the other hand a simple calculation shows that

$$
B=\frac{r^{2}}{8} \int_{-1}^{1} d s \int_{-1}^{1} d t t(\operatorname{sign}(t)-t) \phi^{\prime \prime}\left(X+t \frac{r}{2}\right) \phi^{\prime \prime}\left(X+s t \frac{r}{2}\right) \frac{h^{\prime}(X)}{h^{\prime}\left(X+s t \frac{r}{2}\right)}
$$

which clearly implies

$$
|B| \leqq C r^{2}
$$

on $\tilde{D}$. This together with the estimates (4.9) proves (4.8).

Thus it seems to be a good idea to approximate the operator (4.1) by the simpler one

$$
\begin{aligned}
\hat{\Lambda}_{0}(\omega) f(s)= & p \int_{0}^{\infty} \frac{d \tau}{\tau} \mathbf{e}^{-p(1+\delta) \tau / 2} \int_{-\infty}^{\infty} d s^{\prime} \mathbf{e}^{-(p(1+\delta) / 2 \tau)\left(s-s^{\prime}\right)^{2}+p\left(s-s^{\prime}\right) \eta \circ g(s)} \\
& \cdot\left\{1+i v-(1+\delta) \frac{s-s^{\prime}}{\tau} \eta \circ g(s)\right\} f\left(s^{\prime}\right) .
\end{aligned}
$$

We claim that for $\omega \in \Omega$ the operator $R \equiv \hat{\Lambda}(\omega)-\hat{\Lambda}_{0}(\omega)$ is Hilbert-Schmidt. If we restrict its integral kernel to the complement of $D$ this follows from Lemma 4.1 and its analog for $\hat{\Lambda}_{0}$. We thus consider the kernel of $R$ on the diagonal $D$. Writing

$$
R=\int_{0}^{\infty} V(\tau) d \tau
$$

it suffices to show that the Hilbert-Schmidt norm of the integral kernel of $V(\tau)$ restricted to $D$ is integrable. Direct calculation and use of Lemmas 3.4, 4.2 and 4.3 give an upper bound for this norm as a sum of terms of the following form:

$$
\left\{\tau^{-2 n} \int_{\widetilde{D}} d X d r r^{2 k}\langle X\rangle^{2 j} \mathbf{e}^{-p(1+\operatorname{Re} \delta)\left(\tau+r^{2} \chi^{2}(X, r) / \tau\right)+2 p r \chi(X, r)}\right\}^{1 / 2}
$$

where $\langle X\rangle=\sqrt{1+X^{2}}$, and the integers $k, j$ and $n$ take the values given in the following table:

$\begin{array}{lllllrrrrrr}\mathbf{k} & 4 & 5 & 5 & 6 & 2 & 3 & 3 & 4 & 2 & 3 \\ \mathbf{j} & 0 & 0 & 1 & 1 & -2 & -2 & -1 & -1 & -2 & -2 \\ \mathbf{n} & 1 & 1 & 2 & 2 & 1 & 1 & 2 & 2 & 2 & 2\end{array}$


A simple change of variables in the integral (4.11) further gives the bound

$$
\left\{\tau^{k+1 / 2-2 n} \int_{\mathbb{R}^{2}} d X d r r^{2 k}\langle X\rangle^{-1-2(k-j)} \mathbf{e}^{-p H[r, \sqrt{\tau}]}\right\}^{1 / 2},
$$

where the quadratic form $H$ has the matrix

$$
H \equiv\left(\begin{array}{cc}
(1+\operatorname{Re} \delta) \frac{\chi^{2}(X, r)}{\langle X\rangle^{2}} & \frac{\chi(X, r)}{\langle X\rangle} \\
\frac{\chi(X, r)}{\langle X\rangle} & (1+\operatorname{Re} \delta)
\end{array}\right) \geqq C \operatorname{Re} \delta .
$$

The last inequality, which follows from Lemma 3.4, allows to estimate (4.12) by

$$
C \mathrm{e}^{-C \operatorname{Re} \delta \tau} \tau^{k / 2+1 / 4-n}
$$

with a finite constant $C$ as long as $j \leqq k-1$. This bound is integrable if $n-1<k / 2+1 / 4$ and its integral vanishes like $(\operatorname{Re} \delta)^{-k / 2+n-5 / 4}$ as $\operatorname{Re} \delta \rightarrow \infty$. A look at the above table shows that these two conditions are indeed satisfied. The same argument applies to $\partial_{\omega} R$, thus $R$ is analytic from $\Omega$ to the Hilbert-Schmidt operators on $L^{2}(\mathbb{R})$, and its norm vanishes like $(\operatorname{Re} \delta)^{-1 / 4}$ as $\operatorname{Re} \delta \rightarrow \infty$.

We are now able to compute the (left) symbol of the integral operator $\hat{\Lambda}_{0}$ (we set $\tilde{\eta} \equiv \eta \circ g$ )

$$
\begin{aligned}
& p \int_{0}^{\infty} \frac{d \tau}{\tau} \int_{-\infty}^{\infty} d r \mathbf{e}^{-p(1+\delta)\left(\tau+r^{2} / \tau\right) / 2+p r \tilde{\eta}(s)-i r \xi}\left\{1+i v-(1+\delta) \tilde{\eta}(s) \frac{r}{\tau}\right\} \\
& =\frac{1}{\sqrt{2 \pi p(1+\delta)}} \int_{0}^{\infty} \frac{d \tau}{\sqrt{\tau}} \mathbf{e}^{-\tau\left(p(1+\delta) / 2+(\xi+i p \tilde{\eta})^{2} / 2 p(1+\delta)\right)}\left\{p\left(1-\tilde{\eta}^{2}\right)+i(\omega+\xi \tilde{\eta})\right\} \\
& =\frac{i(\omega+\tilde{\eta} \xi)+p\left(1-\tilde{\eta}^{2}\right)}{\sqrt{\xi^{2}+2 i p(\omega+\tilde{\eta} \xi)+p^{2}\left(1-\tilde{\eta}^{2}\right)}} .
\end{aligned}
$$

One easily checks that this symbol is a bounded continuous function of $s$ and $\xi$, so one expects $\hat{\Lambda}_{0}$ to be a bounded operator. Assuming $\phi \in C^{\infty}$ this follows easily from integration by part, under our more restrictive hypotheses one needs some deeper results (see for example [CM], Chap. 1, Theorem 3), but this remains true. On the other hand a simple computation shows that the symbol $a(x, \xi)$ is in $L^{2}\left(\mathbb{R}^{2}\right)$ if and only if the corresponding operator is Hilbert-Schmidt on $L^{2}(\mathbb{R})$ with norm

$$
\|\mathrm{Op}(a)\|_{L^{2}\left(\mathbb{R}^{2}\right)}^{2}=\int|a(x, \xi)|^{2} d x d \xi .
$$

Thus since $1-\tilde{\eta}^{2}(s)=O\left(s^{-1}\right)$ at infinity we easily check that the terms in $1-\tilde{\eta}^{2}$ in both numerator and denominator of (4.13) may be neglected without changing the result. We just proved the

Theorem 4.4. The formula (4.1) defines an analytic map $\omega \mapsto \hat{\Lambda}(\omega)$ from $\Omega$ to the bounded operators on $L^{2}(\mathbb{R})$. The operator $\hat{\Lambda}(\omega)$ is pseudo-differential with a symbol given by

$$
\lambda(\omega ; s, \xi)=i \frac{(\omega+\xi \tilde{\eta}(s))}{\sqrt{\xi^{2}+2 i p(\omega+\xi \tilde{\eta}(s))}}+r(\omega ; s, \xi)
$$


Moreover $\mathrm{Op}(r)$ is Hilbert-Schmidt and its norm satisfies

$$
\|\mathrm{Op}(r)\|_{L^{2}\left(\mathbb{R}^{2}\right)}=O\left((\operatorname{Re} \delta)^{-1 / 4}\right)
$$

as $\operatorname{Re} \delta$ tends to infinity.

It follows immediately that the operator $\hat{A}(\omega)$ given by (2.17) is well defined, closed and $m$-sectorial on $L^{2}(\mathbb{R})$, with a sector of the type $\left\{z \in \mathbb{C}|| \operatorname{Arg}\left(z-s_{0}\right) \mid<\varphi_{0}<\frac{\pi}{2}\right\}$. Its domain is the Sobolev space $W^{2,2}(\mathbb{R})=\left\{f \in L^{2}(\mathbb{R}) \mid f^{\prime \prime} \in L^{2}(\mathbb{R})\right\}$. Furthermore it defines an analytic family in the sense of Kato (see [RS] or [K]).

\section{The Spectrum of the Operator $\hat{A}(\omega)$}

This section is devoted to the study of the spectral properties of the operator $\hat{A}(\omega)$ that are needed in order to solve the linearized evolution equation (2.15). Loosely speaking we will calculate the continuous spectrum of $\hat{A}(\omega)$ and find a region containing all its eigenvalues. Let us introduce the self-adjoint operator $D \equiv \frac{1}{i} \partial_{s}$ on $L^{2}(\mathbb{R})$. We will say that an operator $T$ on this space is $D^{\alpha}$-bounded (respectively $D^{\alpha}$-compact) if

$$
T\left(1+D^{2}\right)^{-\alpha / 2}
$$

is bounded (respectively compact). Our aim is to further simplify $\hat{A}(\omega)$ by omitting now relatively compact terms. Since a $D^{2}$-compact operator will also be compact relative to any $D$-bounded perturbation of $\sigma D^{2}$, we will in fact calculate modulo $D$-bounded and $D^{2}$-compact errors. The first step is the

Lemma 5.1. Let $1 \equiv \chi_{-}(s)+\chi_{+}(s)$ be a smooth partition of unity on $\mathbb{R}$ such that the sets

$$
\operatorname{supp}\left(\chi_{ \pm}\right) \cap \mathbb{R}_{\mp}
$$

are compact. Then $\hat{A}(\omega)=\hat{A}_{0}(\omega)$ modulo a $D^{2}$-compact error, where

$$
\hat{A}_{0}(\omega) \equiv \sigma D^{2}+\chi_{-}(s) \lambda_{-}(\omega ; D)+\chi_{+}(s) \lambda_{+}(\omega ; D)
$$

and

$$
\lambda_{ \pm}(\omega ; \xi) \equiv i \frac{\omega \pm \xi}{\sqrt{\xi^{2}+2 i p(\omega \pm \xi)}} .
$$

Furthermore the error $E(\omega) \equiv \hat{A}(\omega)-\hat{A}_{0}(\omega)$ is uniformly bounded as $\operatorname{Re} \delta \rightarrow \infty$.

Proof. From (4.15) and (5.1) we see that the error $E$ is a pseudo-differential operator whose symbol is the sum of the three following terms:

$$
\begin{aligned}
& e_{0} \equiv-\sigma \kappa_{0}^{2}(s)-j_{0}^{\perp}(s), \\
& e_{ \pm} \equiv \chi_{ \pm}(s)\left(\lambda(\omega ; s, \xi)-\lambda_{ \pm}(\omega ; \xi)\right) .
\end{aligned}
$$

The first term is bounded by our assumptions and Theorem 3.1, it is further independent of $\omega$. The $D^{2}$-compactness follows from the Rellich criterion since $j_{0}^{\perp}$ and $\kappa_{0}$ vanish at infinity (see $[\mathrm{RS}]$ ). The two remaining terms are $D^{2}$ - 
Hilbert-Schmidt, to see this we use Theorem 4.4 and Taylor's formula to get

$$
e_{+}=-i \chi_{+}(s)(1-\tilde{\eta}(s)) \frac{\xi\left(\xi^{2}+i p(\omega+\xi)\right)}{\left(\xi^{2}+2 i p(\omega+\xi)\right)^{3 / 2}}+\tilde{r}(\omega ; s, \xi)
$$

with the following expression for the remainder

$$
\tilde{r}(\omega ; s, \xi) \equiv \chi_{+}(s) r(\omega ; s, \xi)+\chi_{+}(s)(1-\tilde{\eta}(s))^{2} \int_{0}^{1} \frac{p \xi^{2}\left(2 \xi^{2}+i p\left(\omega+\xi \tilde{\eta}_{t}(s)\right)\right)}{\left(\xi^{2}+2 i p\left(\omega+\xi \tilde{\eta}_{t}(s)\right)\right)^{5 / 2}} d t,
$$

where $\tilde{\eta}_{t}(s) \equiv 1-t(1-\tilde{\eta}(s))$. The first term in (5.2) is easily controlled since it factorizes into a product of a bounded function of $s$ which decays like $s^{-1}$ at infinity, and a uniformly bounded function of $\xi$ and $\omega$. Finally a look at $\tilde{r}$ shows that its $L^{2}$-norm vanishes as $\operatorname{Re} \delta \rightarrow \infty$ (for $r$ this follows from Theorem 4.4), thus we can apply Formula (4.14) for the Hilbert-Schmidt norm of a pseudo-differential operator. Of course an analogous argument applies to $E_{-}$, so the proof is complete.

One important property of the approximant $\hat{A}_{0}$ is that it tends to definite translation invariant limits at spatial infinity. However the fact that the left and right limits

$$
\hat{A}_{\mp}(\omega) \equiv \sigma D^{2}+\lambda_{\mp}(\omega ; D)
$$

do not coincide makes it impossible to further subtract some residual $D^{2}$-compact part. The spectral analysis of the limiting operators $\hat{A}_{ \pm}$is quite trivial, we have

$$
\operatorname{spec}\left(\hat{A}_{-}(\omega)\right)=\operatorname{spec}\left(\hat{A}_{+}(\omega)\right)=\Sigma_{\omega} \equiv\left\{\sigma \xi^{2}+\lambda_{+}(\omega ; \xi) \mid \xi \in \mathbb{R}\right\} .
$$

This curve is easily seen to be asymptotic (at infinity) to the half-lines $\mathbb{R}_{+} \pm i$ as $|\xi| \rightarrow \infty$ (see Fig. 1), so it cuts the complex plane into open and simply connected components $\rho_{\alpha}(\omega)$ one of which, say $\rho_{0}(\omega)$, contains a large sector $\mathbb{C} \backslash\left\{|\operatorname{Arg}(z-s)| \leqq \varphi<\frac{\pi}{2}\right\}$ for some real $s$ and $\varphi$.

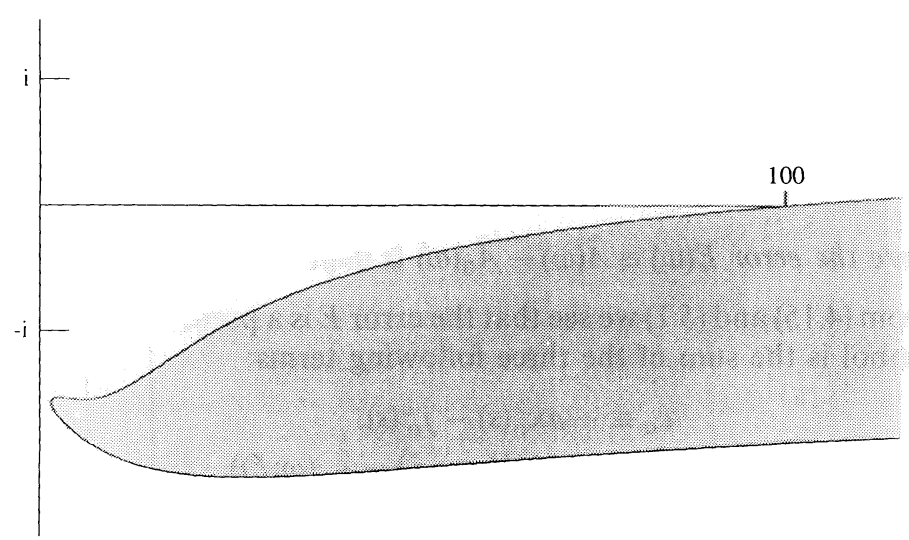

Fig. 1. The curve $\Sigma_{\omega}$ for $\omega=-10 p$ and $\sigma p^{2}=1$, the complement of $\rho_{0}(\omega)$ is dashed 
To disentangle the left and right parts of $\hat{A}_{0}$ we now use a nice tool introduced by Davies and Simon in [DS]: the twisting trick. Denote by $\mathscr{C}$ the (anti-unitary, involutive) complex conjugation on $L^{2}(\mathbb{R})$, and define the transposition of linear operators by

$$
H^{\top} \equiv \mathscr{C} H^{*} \mathscr{C}
$$

Since $\mathscr{C}$ and the hermitian conjugation both (complex)-conjugate the spectrum, transposition clearly leaves it invariant and we conclude that the operator

$$
\hat{\mathscr{A}}(\omega) \equiv\left(\begin{array}{cc}
\hat{A}(\omega) & 0 \\
0 & \hat{A}^{\top}(\omega)
\end{array}\right)
$$

on $L^{2}(\mathbb{R}) \oplus L^{2}(\mathbb{R})$ has the same spectrum than $\hat{A}(\omega)$ (only the multiplicities are modified). A simple calculation shows that

$$
\hat{A}_{0}^{\top}(\omega) \equiv \sigma D^{2}+\lambda_{+}(\omega ; D) \chi_{-}(s)+\lambda_{-}(\omega ; D) \chi_{+}(s) .
$$

Setting $\chi_{+}(s)=\cos ^{2}(\theta(s))$ with $\theta^{\prime} \in C_{0}^{\infty}$ and defining the unitary operator

$$
\mathscr{U} \equiv\left(\begin{array}{cc}
\cos \theta & \sin \theta \\
-\sin \theta & \cos \theta
\end{array}\right)
$$

on $L^{2} \oplus L^{2}$, a little algebra gives

$$
\mathscr{U}\left(\begin{array}{cc}
\hat{A}_{+} & 0 \\
0 & \hat{A}_{-}
\end{array}\right) \mathscr{U}^{-1} \equiv \mathscr{U} \hat{\mathscr{A}}_{0} \mathscr{U}^{-1}=\hat{\mathscr{A}}+\mathscr{K}
$$

with

$$
\mathscr{K} \equiv\left(\begin{array}{cc}
\cos \theta\left[\hat{A}_{+}, \cos \theta\right]+\sin \theta\left[\hat{A}_{-}, \sin \theta\right]-E & -\cos \theta \hat{A}_{+} \sin \theta+\sin \theta \hat{A}_{-} \cos \theta \\
-\sin \theta \hat{A}_{+} \cos \theta+\cos \theta \hat{A}_{-} \sin \theta & {\left[\sin \theta, \hat{A}_{+}\right] \sin \theta+\left[\cos \theta, \hat{A}_{-}\right] \cos \theta-E^{\top}}
\end{array}\right) .
$$

Thus by (5.6) the spectrum of $\hat{\mathscr{A}}+\mathscr{K}$ is nothing but $\Sigma_{\omega}$. We claim that $\mathscr{K}(\omega)$ is $D^{2}$-compact and uniformly $D$-bounded as $\operatorname{Re} \delta \rightarrow \infty$. Postponing the proof of this assertion let us show how it allows to get the spectrum of $\hat{A}(\omega)$ under control.

Since there is no uniquely and widely accepted definition of the essential spectrum (especially for non-self-adjoint operators, see in [LM] the article of K. Gustafson for a review of all essential spectra used in the literature and their inter-connections), we first give a precise meaning to this concept. For a closed operator $L$ defined on a domain $D(L)$ of a Hilbert space $\mathscr{H}$, the discrete spectrum is the set $\sigma_{d}(L)$ of all its isolated eigenvalues of finite multiplicity. We will call essential spectrum of $L$ the complementary set $\sigma_{\text {ess }}(L) \equiv \operatorname{spec}(L) \backslash \sigma_{d}(L)$. In our case this clearly gives

$$
\begin{gathered}
\sigma_{\text {ess }}\left(\hat{A}_{ \pm}(\omega)\right)=\sigma_{\text {ess }}(\hat{\mathscr{A}}(\omega)+\mathscr{K}(\omega))=\Sigma_{\omega}, \\
\sigma_{d}\left(\hat{A}_{ \pm}(\omega)\right)=\sigma_{d}(\hat{\mathscr{A}}(\omega)+\mathscr{K}(\omega))=\varnothing .
\end{gathered}
$$

We further recall that a sequence $u_{n}$ in $\mathscr{H}$ is said to be characteristic for the operator $L$ if it satisfies:

(i) $u_{n} \in D(L)$ for all $n$,

(ii) $\liminf _{n \rightarrow \infty}\left\|u_{n}\right\|>0$, 
(iii) $w$ - $\lim _{n \rightarrow \infty} u_{n}=0 \quad$ (weak convergence in $\mathscr{H}$ ),

(iv) $\lim _{n \rightarrow \infty} L u_{n}=0$ (norm convergence in $\mathscr{H}$ ).

We call limit-spectrum of $L$ the set

$$
W(L) \equiv\{z \in \mathbb{C} \mid(L-z) \text { has a characteristic sequence }\}
$$

which has a variety of other names in the literature (see [S], Chap. 11, where it is denoted by $\sigma_{e \alpha}$ ). The main interest of this definition is Weyl's criterion (see [HV] Theorem 2.1) which tells us that

(1) $W(L)$ is closed,

(2) $W(L) \subset \sigma_{\text {ess }}(L)$,

(3) $\partial \sigma_{\text {ess }}(L) \subset W(L)$,

and the fact that the limit-spectrum is invariant under relatively compact perturbations (see [S.]). From (5.7), (2) and (3) we clearly get

$$
W(\hat{\mathscr{A}}(\omega)+\mathscr{K}(\omega))=\Sigma_{\omega} .
$$

Now we claimed that $\mathscr{K}$ is $D^{2}$-compact, thus the stability of the limit-spectrum and Weyl's criterion (2) give

$$
W(\hat{\mathscr{A}}(\omega))=\Sigma_{\omega} \subset \sigma_{\text {ess }}(\hat{\mathscr{A}}(\omega)) .
$$

Consider now any of the connected components $\rho_{\alpha}$ of the complex plane, Weyl's criterion (3) implies that it either belongs entirely to $\sigma_{\text {ess }}(\hat{\mathscr{A}})$ or is completely disjoint from it i.e., only contains discrete spectrum. One of this set, namely $\rho_{0}$, clearly intersect the resolvent set of $\hat{\mathscr{A}}$ and thus cannot be in the essential spectrum. What happens to the other components is not clear but fortunately also irrelevant for our purpose. According to the identity of the spectra of $\hat{\mathscr{A}}$ and $\hat{A}$ the same conclusions hold for the later, thus

$$
\begin{gathered}
\partial \rho_{0}(\omega) \subset \Sigma_{\omega} \subset \sigma_{\text {ess }}(\hat{A}(\omega)), \\
\rho_{0}(\omega) \cap \sigma_{\text {ess }}(\hat{A}(\omega))=\varnothing .
\end{gathered}
$$

To localize the discrete spectrum we need some bounds on the resolvent of the non-perturbed operator $\hat{\mathscr{A}}_{0}$. From the functional calculus of self-adjoint operators we get

$$
\left\|\left(1+D^{2}\right)^{\alpha / 2}\left(\hat{\mathscr{A}}_{0}(\omega)-z\right)^{-1}\right\|=\sup _{\xi \in \mathbb{R}}\left|\frac{\left(1+\xi^{2}\right)^{\alpha / 2}}{\sigma \xi^{2}+\lambda_{+}(\omega ; \xi)-z}\right| .
$$

For $\alpha=0$ and $z \in \rho_{0}(\omega)$ this clearly means

$$
\left\|\left(\hat{\mathscr{A}}_{0}(\omega)-z\right)^{-1}\right\|=\frac{1}{\operatorname{dist}\left(z, \Sigma_{\omega}\right)} .
$$

We will prove in the next section (in Lemma 6.1) that if $\operatorname{Re} \delta$ is large enough, depending only of $\sigma p^{2}$, then $\operatorname{Re}\left(\sigma \xi^{2}+\lambda_{+}\right)>0$. For $0<\alpha \leqq 2$ this allows to get the 
estimate

$$
\begin{aligned}
\left|\frac{\left(1+\xi^{2}\right)^{\alpha / 2}}{\sigma \xi^{2}+\lambda_{+}(\omega ; \xi)-z}\right| & \leqq\left|\frac{1+\xi^{2}}{\sigma \xi^{2}+\lambda_{+}(\omega ; \xi)-z}\right|^{\alpha / 2} \frac{1}{\operatorname{dist}\left(z, \Sigma_{\omega}\right)^{1-\alpha / 2}} \\
& \leqq \frac{C}{\operatorname{dist}\left(z, \Sigma_{\omega}\right)^{1-\alpha / 2}}
\end{aligned}
$$

for some constant $C$ if $z \in \rho_{0}(\omega)$ and $\operatorname{Re} z \leqq 0$. The claimed uniform $D$-boundedness of $\mathscr{K}$, which clearly implies that of $\widehat{\mathscr{K}} \equiv \mathscr{U}^{-1} \mathscr{K} \mathscr{U}$ (since $\mathscr{U}$ is smooth), allows to apply a standard perturbative argument (see [K], Chap. IV, Theorem 3.17 for example) based on the resolvent formula associated to Eq. (5.6)

$$
(\hat{\mathscr{A}}-z)^{-1}=\mathscr{U}\left(\hat{\mathscr{A}}_{0}-z\right)^{-1}\left(1-\tilde{\mathscr{K}}\left(\hat{\mathscr{A}}_{0}-z\right)^{-1}\right)^{-1} \mathscr{U}^{-1}
$$

to get the

Theorem 5.2. Let $\Sigma_{\omega}$ be the curve defined by (5.4) and $\rho_{0}(\omega)$ the large connected component of $\mathbb{C} \backslash \Sigma_{\omega}$ (i.e., that containing a half-plane of the form $\left\{\operatorname{Re} z<r_{0}\right\}$ ). Then for $\omega \in \Omega$ we have

$$
\begin{gathered}
\partial \rho_{0}(\omega) \subset \Sigma_{\omega} \subset \sigma_{\text {ess }}(\hat{A}(\omega)), \\
\rho_{0}(\omega) \cap \sigma_{\text {ess }}(\hat{A}(\omega))=\varnothing,
\end{gathered}
$$

whereas on any set $\{\omega \in \Omega \mid \operatorname{Re} \delta \geqq \varepsilon>0\}$ the discrete spectrum satisfies

$$
\operatorname{dist}\left(\sigma_{d}(\hat{A}(\omega)), \Sigma_{\omega}\right) \leqq d_{\varepsilon}<\infty
$$

for some constant $d_{\varepsilon}$.

Furthermore if $\varepsilon$ is large enough, then for any $z \in \rho_{0}(\omega) \cap\{z \mid \operatorname{Re} z \leqq 0\}$ such that $\operatorname{dist}\left(z, \Sigma_{\omega}\right)>d_{\varepsilon}$ the estimate

$$
\left\|\left(1+D^{2}\right)^{\alpha / 2}(\hat{\mathscr{A}}(\omega)-z)^{-1}\right\| \leqq \frac{C}{\operatorname{dist}\left(z, \partial \rho_{0}(\omega)\right)^{1-\alpha / 2}}
$$

holds for some constant $C$ and any $0 \leqq \alpha \leqq 2$.

Turning to the proof of our claim, we first rewrite the operator $\mathscr{K}$ as a sum of three terms

$$
\begin{aligned}
& \mathscr{K}_{1} \equiv\left(\begin{array}{cc}
-E & 0 \\
0 & -E^{\top}
\end{array}\right), \\
& \mathscr{K}_{2} \equiv\left(\begin{array}{cc}
0 & -\cos \theta \sin \theta\left(\hat{A}_{+}-\hat{A}_{-}\right) \\
-\sin \theta \cos \theta\left(\hat{A}_{+}-\hat{A}_{-}\right) & 0
\end{array}\right), \\
& \mathscr{K}_{3} \equiv\left(\begin{array}{cc}
\cos \theta\left[\hat{A}_{+}, \cos \theta\right]+\sin \theta\left[\hat{A}_{-}, \sin \theta\right] & -\cos \theta\left[\hat{A}_{+}, \sin \theta\right]+\sin \theta\left[\hat{A}_{-}, \cos \theta\right] \\
-\sin \theta\left[\hat{A}_{+}, \cos \theta\right]+\cos \theta\left[\hat{A}_{-}, \sin \theta\right] & {\left[\sin \theta, \hat{A}_{+}\right] \sin \theta+\left[\cos \theta, \hat{A}_{-}\right] \cos \theta}
\end{array}\right) .
\end{aligned}
$$

For the first term $\mathscr{K}_{1}$ the claim already follows from Lemma 5.1, to handle the second we simply remark that the support of $\sin (\theta) \cos (\theta)$ is compact and that $\left(\hat{A}_{+}-\hat{A}_{-}\right)$is a convolution operator with a uniformly bounded symbol, thus $\mathscr{K}_{2}$ is $D^{2}$-compact and uniformly bounded. Finally for $\mathscr{K}_{3}$ we only need to control 
the commutators

$$
\left[\hat{A}_{ \pm}, \mathrm{e}^{i \varepsilon \theta(s)}\right]=\sigma\left[D^{2}, \mathrm{e}^{i \varepsilon \theta(s)}\right]+\left[\lambda_{ \pm}(\omega ; D), \mathrm{e}^{i \varepsilon \theta(s)}\right],
$$

for $\varepsilon= \pm 1$. The first term in the right-hand side can be explicitly computed, it is independent of $\omega, D^{2}$-compact and $D$-bounded (recall that $\theta^{\prime} \in C_{0}^{\infty}$ ). The second commutator is in fact uniformly Hilbert-Schmidt as a direct application of the following lemma shows. Thus the claim is proved.

Lemma 5.3. Let the two functions $\chi(s)$ and $\lambda(\xi)$ have first derivatives in $L^{2}(\mathbb{R})$, then the commutator

$$
C \equiv[\chi(s), \lambda(D)]
$$

is Hilbert-Schmidt on $L^{2}$ with a norm bounded by

$$
\|C\|_{\mathrm{HS}} \leqq\left\|\chi^{\prime}\right\|_{L^{2}}\left\|\lambda^{\prime}\right\|_{L^{2}} \text {. }
$$

Proof. The distributional kernel of $C$ is easily calculated, it is given by

$$
C(x, y) \equiv(\chi(x)-\chi(y)) \hat{\lambda}(x-y)=i\left(\frac{\chi(x)-\chi(y)}{x-y}\right)\left(\lambda^{\prime}\right)^{\wedge}(x-y)
$$

so that we can write

$$
C=\frac{1}{2} \int_{-1}^{1} K_{t} d t
$$

where the operator $K_{t}$ has the integral kernel

$$
K_{t}\left(X+\frac{r}{2}, X-\frac{r}{2}\right) \equiv \chi^{\prime}\left(X+t \frac{r}{2}\right)\left(\lambda^{\prime}\right)^{\wedge}(r) .
$$

Since the Hilbert-Schmidt norm of $K_{t}$ can be explicitly evaluated

$$
\left\|K_{t}\right\|_{\mathrm{HS}}=\left\|\chi^{\prime}\right\|_{L^{2}}\left\|\lambda^{\prime}\right\|_{L^{2}}
$$

the result follows by integrating over $t$.

\section{Solving the Linear Equation}

In this section we construct the solution of the linear perturbation equation (2.15) by solving for $\hat{f}$ the relation (2.17) and Fourier transforming back to the time variable. To do this we clearly have to invert the operator $\hat{A}(\omega)$ i.e., we need $0 \notin \operatorname{spec}(\hat{A}(\omega))$. Let us first look at the weaker condition $0 \notin \sigma_{\text {ess }}(\hat{A}(\omega))$. The needed estimate of the symbol of $\hat{A}_{+}$is achieved by the following

Lemma 6.1. There is a positive constant $R$ depending only on $\sigma p^{2}$ such that the lower bound

$$
\operatorname{Re}\left(\sigma \xi^{2}+\lambda_{+}(\omega ; \xi)\right) \geqq \frac{\operatorname{Re} \delta}{5}
$$

holds provided $\operatorname{Re} \delta>R$. 
Proof. By rescaling $\xi$ and $\sigma$ we may always assume that $p=1$. Let us denote by $w$ the square root that occurs in the denominator of $\lambda_{+}$, then we can rewrite

$$
\operatorname{Re} \lambda_{+}=\frac{1}{2} \operatorname{Re} w\left(1-\frac{\xi^{2}}{|w|^{2}}\right)
$$

from which we get the bound

$$
\operatorname{Re}\left(\sigma \xi^{2}+\lambda_{+}(\omega ; \xi)\right) \geqq\left(\sigma-\frac{1}{2|w|}\right) \xi^{2}+\frac{1}{2} \operatorname{Re} w .
$$

But the identity

$$
w \equiv \sqrt{\xi^{2}+2 i(\omega+\xi)}=\sqrt{(\xi-i \delta)(\xi+2 i+i \delta)}
$$

leading to $|w|>\operatorname{Re} \delta$, we further have

$$
\operatorname{Re}\left(\sigma \xi^{2}+\lambda_{+}(\omega ; \xi)\right) \geqq \frac{1}{2}\left(\sigma \xi^{2}+\operatorname{Re} w\right)
$$

for sufficiently large $\operatorname{Re} \delta$. We now distinguish two cases, first we assume

$$
\left|\frac{i+\xi}{1+\delta}\right|<\frac{1}{\sqrt{2}}
$$

and rewrite the square root in $\lambda_{+}$as

$$
w=\sqrt{(i+\xi)^{2}+(1+\delta)^{2}}=(1+\delta)(1+\varepsilon) \quad \text { with } \quad|\varepsilon|<\left|\frac{i+\xi}{1+\delta}\right|^{2}<\frac{1}{2} .
$$

From this formula we easily get the estimate

$$
\begin{aligned}
\operatorname{Re} w & \geqq \frac{1}{2} \operatorname{Re} \delta-|\operatorname{Im} \delta||\varepsilon| \\
& \geqq \frac{1}{2} \operatorname{Re} \delta-\frac{|\operatorname{Im} \delta|}{|1+\delta|} \frac{|i+\xi|}{\sqrt{2}} \\
& \geqq \frac{1}{2} \operatorname{Re} \delta-|i+\xi| .
\end{aligned}
$$

By (6.3) this clearly leads to

$$
\begin{aligned}
\operatorname{Re}\left(\sigma \xi^{2}+\lambda_{+}(\omega ; \xi)\right) & \geqq \frac{1}{2}\left(\sigma \xi^{2}-|i+\xi|+\frac{1}{2} \operatorname{Re} \delta\right) \\
& \geqq \frac{\operatorname{Re} \delta}{5}
\end{aligned}
$$

for sufficiently large $\operatorname{Re} \delta$. In the opposite case we have

$$
\left|\frac{i+\xi}{1+\delta}\right|>\frac{1}{\sqrt{2}}
$$

from which we get

$$
|i+\xi| \geqq \frac{\operatorname{Re} \delta}{\sqrt{2}},
$$


and thus by Theorem 5.2 and the fact that $\operatorname{Re} w \geqq 0$

$$
\operatorname{Re}\left(\sigma \xi^{2}+\lambda_{+}(\omega ; \xi)\right) \geqq \frac{1}{2} \sigma \xi^{2} \geqq \frac{1}{2} \sigma(\operatorname{Re} \delta)^{2}-1 \geqq \frac{\operatorname{Re} \delta}{5}
$$

for large $\operatorname{Re} \delta$, this complete the proof.

This lemma shows that if $\operatorname{Re} \delta$ is large the point 0 belongs to $\rho_{0}(\omega)$ and, by Theorem 5.2, it is not in the essential spectrum of $\hat{A}(\omega)$. This remains true as $\omega$ varies in $\Omega$ as long as $\Sigma_{\omega}$ does not cross the origin. Thus the connected component $\Omega_{F}$ of $\left\{\omega \in \Omega \mid 0 \notin \Sigma_{\omega}\right\}$ containing a half-plane $\{\operatorname{Im} \omega<c\}$ has the properties

$$
\begin{aligned}
\omega \in \Omega_{F} & \Rightarrow 0 \notin \sigma_{\text {ess }}(\hat{A}(\omega)), \\
\omega \in \partial \Omega_{F} \backslash \partial \Omega & \Rightarrow 0 \in \sigma_{\text {ess }}(\hat{A}(\omega)) .
\end{aligned}
$$

Next we consider the condition $0 \notin \sigma_{d}(\hat{A}(\omega))$. The lower bound (6.1) together with (5.10) imply that if $\operatorname{Re} \delta$ is large enough the discrete spectrum of $\hat{A}(\omega)$ is uniformly bounded away from 0 . Thus if $R$ is large enough the operators $\hat{A}(\omega)^{-1}$ and therefore $\hat{\mathscr{A}}(\omega)^{-1}$ exist and are analytic in the region $\{\omega \in \Omega \mid \operatorname{Re} \delta>R\}$. Furthermore by Eq. (5.8) the resolvent formula

$$
\hat{\mathscr{A}}^{-1}=\mathscr{U} \hat{\mathscr{A}}_{0}^{-1}\left(1-\tilde{\mathscr{K}} \hat{\mathscr{A}}_{0}^{-1}\right)^{-1} \mathscr{U}^{-1}
$$

holds in this region. Since $\tilde{\mathscr{K}} \hat{\mathscr{A}}_{0}^{-1}$ is compact by the results of Sect. 5, we may apply analytic Fredholm theory (see [RS]) to conclude that $\hat{\mathscr{A}}^{-1}$ and thus also $\hat{A}^{-1}$ are in fact meromorphic in the domain $\Omega_{F}$ with poles at the values of $\omega$ for which $0 \in \sigma_{d}(\hat{A}(\omega))$ and finite rank residues there. Thus we have the

Theorem 6.2. The inverse operator $\hat{F}(\omega) \equiv \hat{A}(\omega)^{-1}$ exists as a meromorphic function from $\Omega_{F}$ to the bounded Fredholm operators on $L^{2}(\mathbb{R})$. Its poles are all located within the region $\{\omega \in \Omega \mid \operatorname{Re} \delta<R\}$ for some constant $R$, and have finite rank residues. Furthermore the bound

$$
\left\|\left(1+D^{2}\right)^{\alpha / 2} \hat{F}(\omega)\right\| \leqq \frac{C}{(\operatorname{Re} \delta)^{1-\alpha / 2}}
$$

holds outside of this region.

Before turning to Eq. (2.17) let us introduce some (Hilbert) function spaces that we will need. For any real $c$ we define

$$
L_{c}^{2}\left(\mathbb{R}_{+}\right) \equiv\left\{f \in L_{\text {loc }}^{2}\left(\mathbb{R}_{+}\right) \mid \mathbf{e}^{c t} f(t) \in L^{2}\left(\mathbb{R}_{+}\right)\right\}
$$

with the obvious norm. The Fourier transform is a unitary map from this space into the Hardy space

$$
H_{2}\left(\mathbb{C}_{c}\right) \equiv\left\{g \mid g \text { is analytic in } \mathbb{C}_{c} \text { and } \sup _{\eta<c} \int_{-\infty}^{\infty}|g(\omega+i \eta)|^{2} d \omega<\infty\right\},
$$

where $\mathbb{C}_{c} \equiv\{z \mid \operatorname{Im} z<c\}$ and the norm is also obvious (see [SW] for example). Finally we define the associated scale of Sobolev spaces

$$
W_{c}^{\alpha, 2}\left(\mathbb{R}_{+}\right) \equiv\left\{f \in L_{c}^{2}\left(\mathbb{R}_{+}\right) \mid \mathbf{e}^{c t} f(t) \in W^{\alpha, 2}(\mathbb{R})\right\}
$$


where $W^{\alpha, 2}$ is the usual Hilbert space

$$
W^{\alpha, 2}(\mathbb{R}) \equiv\left\{f \in L^{2} \mid\left(1+\xi^{2}\right)^{\alpha / 2} \hat{f}(\xi) \in L^{2}\right\} .
$$

For simplicity we shall assume that the perturbation $q$ satisfies

$$
q \in W_{c}^{\alpha, 2}\left(\mathbb{R}_{+}, d t\right) \otimes W^{\beta, 2}(\mathbb{R}, d s)
$$

for some real $\alpha \geqq 0, \beta \geqq 0$ and $c \geqq 0$. Its Fourier transform

$$
\hat{q}(\omega)=\int_{-\infty}^{\infty} q(t) \mathbf{e}^{-i \omega t} d t
$$

is then analytic in the half-plane $\mathbb{C}_{c}$ and satisfies the bound

$$
\int\left(1+\omega^{2}\right)^{\alpha}\|\hat{q}(\omega+i \tilde{c})\|_{W^{\beta, 2}}^{2} d \omega<\infty
$$

for any $\tilde{c} \leqq c$. It follows that $\hat{F}(\omega) \hat{q}(\omega)$ is analytic in $\mathbb{C}_{c_{d}}$, where

$$
c_{d} \equiv \sup \left\{\tilde{c}<c \mid \hat{F} \text { is analytic in } \mathbb{C}_{\tilde{c}}\right\}
$$

and by (6.4) its inverse Fourier transform

$$
f(t)=\int_{i \tilde{c}-\infty}^{i \tilde{c}+\infty} \hat{F}(\omega) \hat{q}(\omega) \mathbf{e}^{i \omega t} d \omega=\mathbf{e}^{-\tilde{c} t} \int_{-\infty}^{\infty} \hat{F}(\omega+i \tilde{c}) \hat{q}(\omega+i \tilde{c}) \mathbf{e}^{i \omega t} d \omega
$$

exists and satisfies

$$
f \in \bigcap_{\gamma<c_{d}} \bigcap_{0 \leqq \varepsilon \leqq 2} W_{\gamma}^{\alpha+1 / 2-\varepsilon / 4,2}\left(\mathbb{R}_{+}\right) \otimes W^{\beta+\varepsilon, 2}(\mathbb{R}) .
$$

By construction this is the unique causal solution of the linearized evolution equation (2.15) in this space. If we now define

$$
c_{\text {ess }}=\sup \left\{\tilde{c}<c \mid \hat{F} \text { is meromorphic with finite rank residues in } \mathbb{C}_{\tilde{c}}\right\},
$$

then for any $\tilde{c}$ between $c_{\text {ess }}$ and $c_{d}$ Cauchy's theorem allows us to deform the integration contour in (6.5) to the line $\{\operatorname{Im} \omega=\tilde{c}\}$ plus small circles around the poles of $\hat{F}(\omega)$ in the strip $\left\{c_{d} \leqq \operatorname{Im} \omega<\tilde{c}\right\}$. Each of this pole $\omega_{j}$ contributes a term

$$
f_{j}(t)=\mathbf{e}^{i \omega_{j} t} f_{j} \equiv \mathbf{e}^{i \omega_{j} t} M_{j} \hat{q}\left(\omega_{j}\right)
$$

to $f(t)$. Here $M_{j}$ is the finite rank residue of $\hat{F}$ at $\omega_{j}$, thus $f_{j}$ is well localized in space uniformly in $q$ in the sense of Ruelle (see [R]). The remaining integral which we call $f_{\tilde{c}}(t)$ then satisfies

$$
f_{\tilde{c}} \in \bigcap_{\gamma<\tilde{c} 0 \leqq} \bigcap_{0 \leqq 2} W_{\gamma}^{\alpha+1 / 2-\varepsilon / 4,2}\left(\mathbb{R}_{+}\right) \otimes W^{\beta+\varepsilon, 2}(\mathbb{R}) .
$$

Tip-splitting terms of the form (6.6) can be unstable, unfortunately they are very difficult to control and should probably be computed numerically. On the other hand the rate of exponential growth of the more interesting side-branching modes is given by $c_{\text {ess }}$ which is much easier to compute, we only need to solve

$$
\sigma \xi^{2}+\lambda_{+}(\omega ; \xi)=0
$$

for $\omega$. Using the quantity $w$ introduced in the proof of Lemma 6.1 we can rewrite 


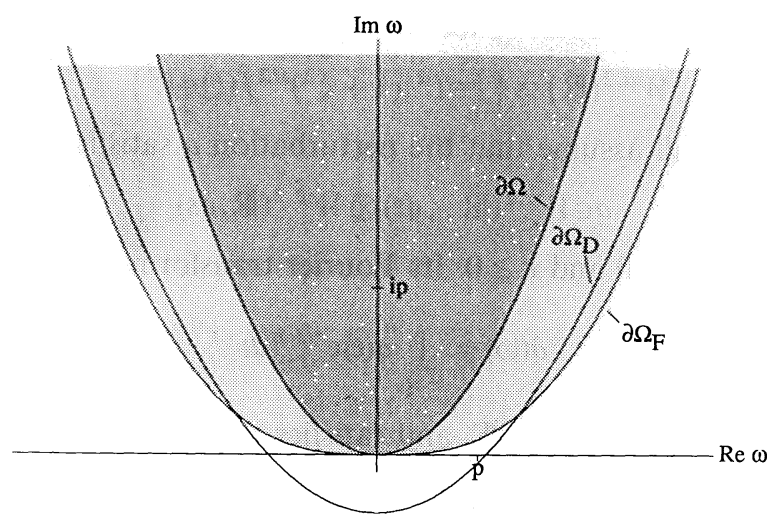

Fig. 2. The $\omega$-plane in the case $\sigma p^{2}=0.1, \hat{A}(\omega)$ is analytic outside the dark grey region and Fredholm outside the light grey one. $\hat{F}(\omega)$ is meromorphic below $\partial \Omega_{F}$, and has no poles below $\partial \Omega_{D}$

this equation as

$$
w^{2}+2 \sigma p \xi^{2} w-\xi^{2}=0 \quad \text { with } \quad \operatorname{Re} w \geqq 0 .
$$

Solving this quadratic equation further gives

$$
w=\sqrt{\sigma^{2} p^{2} \xi^{4}+\xi^{2}}-\sigma p \xi^{2}
$$

which after squaring and comparison with the definition (6.2) finally leads to

$$
\omega=-\xi+i \sigma|\xi|^{3}\left(\sqrt{1+\sigma^{2} p^{2} \xi^{2}}-\sigma p|\xi|\right) \text {. }
$$

This relation should be interpreted as a dispersion relation at infinity, since it expresses the relation between the frequency $\omega$ and the wave number $\xi$ in the limit $s \rightarrow \infty$. A look at Fig. 2 shows that in fact $c_{\text {ess }}=0$, thus the needle-crystals are linearly indifferent against side-branching modes. Note however that the contact of the essential spectrum $\Omega_{F}$ with the real axis is cubic, a fact that should be relevant for the dynamics of the side-branching modes.

\section{Conclusion}

We tried to convince the reader that the calculation of the symbol of an integral operator may be a valuable tool, especially when it is possible to get a more or less explicit formula (this is almost always the case, at least in some asymptotic sense, by the use of stationary phase techniques). In fact, the major use of this pseudo-differential representation was done in the introduction, where we found the right canonical coordinate transformation to simplify the symbol. This step is of fundamental importance since it also selects the function space in which further work is done. In cases where the physical interpretation does not fix this space, the choice is generally very intricate and the insight given by the symbol may be very helpful. Note however that we did not take full advantage of the situation since we have not used any symbolic calculus. The reason is that we did not find the 
appropriate symbol classes in the mathematical literature. There usually no global control on the $x$-variable is available. Since the development of a symbolic calculus would have cost too much space, we decided to use a more pedestrain but far less general approach (this is particularly true of Sect. 4 where a kind of Egorov theorem should have been proved). We stress however that a symbolic calculus exists and we hope it will allow, in the future, to get more detailed information on the solutions of the perturbation equation (2.15), much in the spirit of the WKB-like technique used by Langer in the study of noisy dendrites (see (LP, L3 or BBL]).

Acknowledgements. I am grateful to J.-P. Eckmann and P. Collet for introducing me to the problem and for helpful discussions.

\section{References}

[AS] Abramovitz, M., Stegun, I.: Handbook of Mathematical Functions. New York: Dover 1965

[BBL] Barber, M., Barbieri, A., Langer, J. S.: Dynamics of dentritic sidebranching in the two-dimensional symmetric model of solidification. Phys. Rev. A36, 3340 (1987)

[BGLS1] Ben-Jacob, E., Goldenfeld, N., Langer, J. S., Schoen, G.: Dynamics of interfacial pattern formation. Phys. Rev. Lett. 51, 1930 (1983)

[BGLS2] Ben-Jacob, E., Goldenfeld, N., Langer, J. S., Schoen, G.: Boundary-layer model of pattern formation in solidification. Phys. Rev. A29, 330 (1984)

[BHL] Barbieri, A., Hong, D. C., Langer, J. S.: Velocity selection in the symmetric model of dentritic crystal growth. Phys. Rev. A35, 1802 (1987)

[BKKL1] Brower, R. C., Kessler, D. A., Koplik, J., Levine, H.: Geometrical approach to moving-interface dynamics. Phys. Rev. Lett 51, 1111 (1983)

[BKKL2] Brower, R. C., Kessler, D. A., Koplik, J., Levine, H.: Geometrical model of interface evolution. Phys. Rev. A29, 1335 (1984)

[BL] Barbieri, A., Langer, J. S.: Prediction of dentritic growth rates in the linearized solvability theory. Phys. Rev. A39, 5314 (1989)

[BP] Ben Amar, M., Pomeau, Y.: Theory of dentritic growth in weakly undercooled melt. Europhys. Lett. 2, 307 (1986)

[CCMR] Caroli, B., Caroli, C., Misbah, C., Roulet, B.: On velocity selection for needle-crystals in a fully non-local model of solidification. J. Phys. 48, 547 (1987)

[CCRL] Caroli, B., Caroli, C., Roulet, B., Langer, J. S.: Solvability condition for needle crystals at large undercooling in a nonlocal model of solidification. Phys. Rev. A33, 442 (1986)

[CE] Collet, P., Eckmann, J.-P.: Instabilities and fronts for extended systems. Princeton, NJ: Princeton University Press 1990

[CM] Coifman, R., Meyer, Y.: Au-delà des opérateurs pseudo-différentiels. Soc. Math. de France, Astérisque 57 (1978)

[DS] Davies, E. B., Simon, B.: Scattering theory for systems with different spatial asymptotics on the left and right. Commun. Math. Phys 63, 277 (1978)

[EM] Müller-Krumbhaar, H., Van Der Eerden, J. P.: Sidebranching spectrum for a dendrite model in three dimension. Acta Metall. 34, 839 (1986)

[ER] Erdélyi, A.: Tables of integral transforms I. New York: McGraw-Hill 1954

[GSA] Glicksman, M. E., Schaefer, R. J., Ayers, J. D.: Dentritic growth, a test of theory. Metall. Trans. A7, 1747 (1976)

[H'G1] Huang, S. C., Glicksman, M. E.: Fundamentals of dentritic solidification 1, steady state tip growth. Acta Metall. 29, 701 (1981)

[HG2] Huang, S. C., Glicksman, M. E.: Fundamentals of dentritic solidification 2, development of side branch structure. Acta Metall. 29, 717 (1981)

[HV] Hunziker, W. Vock, E.: Stability of Schrödinger eigenvalue problems. Commun. Math. Phys. 83, 281 (1982)

[I] Ivantsov, G. P.: Dokl. Akad. Nauk. SSSR 58, 567 (1947) 
[K] Kato, T.: Perturbation theory for linear operators. Berlin, Heidelberg, New York: Springer 1984

[KKL1] Kessler, D. A., Koplik, J., Levine, H.: Steady-state dentritic crystal growth. Phys. Rev. A33, 3352 (1986)

[KKL2] Kessler, D. A., Koplik, J., Levine, H.: Pattern selection in fingered growth phenomena. Adv. Phys. 37, 255 (1988)

[KL] Kessler, D. A., Levine, H.: Stability of dentritic crystals. Phys. Rev. Lett. 57, 3069 (1986)

[L1] Langer, J. S.: Instabilities and pattern formation in crystal growth. Rev. Mod. Phys. 52, $1(1980)$

[L2] Langer, J. S.: Lectures in the Theory of Pattern Formation. Proceedings of the Les Houches Summer School, Chance and Matter. Souletie, J., Vannimenus, J., Stora, R. (eds.). Amsterdam: North-Holland 1987

[L3] Langer, J. S.: Dentritic sidebranching in the three-dimensional symmetric model in the presence of noise. Phys. Rev. A36, 3350 (1987)

[LM] LaVita, J. A., Marchand, J. -P.: Scattering theory in mathematical physics. Dordrecht-Holland (1974)

[LM1] Langer, J. S., Müller-Krumbhaar, H.: Theory of dentritic growth 2, instabilities in the limit of vanishing surface tension. Acta Metall. 26, 1689 (1978)

[LM2] Langer, J. S., Müller-Krumbhaar, H.: Theory of dentritic growth 3, effects of surface tension. Acta Metall. 26, 1697 (1978)

[LM3] Müller-Krumbhaar, H., Langer, J. S.: Sidebranching instabilities in a two-dimensional model of dentritic solidification. Acta Metall. 29, 145 (1981)

[LP] Pieters, R., Langer, J. S.: Noise-driven sidebranching in the boundary-layer model of dentritic solidification. Phys. Rev. Lett. 56, 1948 (1986)

[LT] Langer, J. S., Turski, L. A.: Studies in the theory of interfacial stability 1, stationary symmetric model. Acta Metall. 25, 1113 (1977)

[M] Meiron, D. I.: Selection of steady states in the two dimensional symmetric model of dentritic growth. Phys. Rev. A33, 2704 (1986)

[MS1] Mullins, W. W., Sekerka, R. F.: Morphological stability of particle growing by diffusion or heat flow. J. Appl. Phys. 34, 323 (1963)

[MS2] Mullins, W. W., Sekerka, R. F.: Stability of a planar interface during solidification of a dilute binary alloy. J. Appl. Phys. 35, 444 (1964)

[R] Ruelle, D.: A remark on bound states in potential scattering theory. Nuovo Cimento A61, 655 (1969)

[RS] Reed, M., Simon, B.: Analysis of operators. New York: Academic Press 1978

[S] Schechter, M.: Spectra of partial differential operators. Amsterdam: North-Holland 1971

[SGM1] Saito, Y., Goldbeck-Wood, G., Müller-Krumbhaar, H.: Dentritic crystallization: Numerical study of the one-sided model. Phys. Rev. Lett. 58, 1541 (1987)

[SGM2] Saito, Y., Goldbeck-Wood, G., Müller-Krumbhaar, H.: Numerical simulation of dentritic growth. Phys. Rev. A38, 2148 (1988)

[SW] Stein, E. M., Weiss, G.: Introduction to Fourier analysis on Euclidean spaces. Princeton, NJ: Princeton University Press 1971

[VSW] Van Saarloos, W., Weeks, J. D.: Boundary-layer formulation of dentritic growth: Existence of a family of steady-state needle solutions. Phys. Rev. Lett. 55, 1685 (1985) 